\title{
UBVRIZ LIGHT CURVES OF 51 TYPE II SUPERNOVAE
}

\author{
Lluís Galbany $^{1,2}$, Mario Hamuy ${ }^{1,2}$, Mark M. Phillips ${ }^{3}$, Nicholas B. Suntzeff ${ }^{4,5}$, José Maza ${ }^{2}$, Thomas de Jaeger ${ }^{1,2}$, \\ Tania Moraga $^{1,2}$, Santiago González-Gaitán ${ }^{1,2}$, Kevin Krisciunas ${ }^{6}$, Nidia I. Morrell ${ }^{3}$, Joanna Thomas-Osip ${ }^{3}$, \\ Wojtek Krzeminski ${ }^{7}$, Luis GonzÁlez ${ }^{2}$, Roberto AnteZana ${ }^{2}$, Marina Wishnjewski ${ }^{2,27}$, Patrick McCarthy ${ }^{8}$, \\ Joseph P. Anderson ${ }^{9}$, Claudia P. Gutiérrez ${ }^{1,2,9}$, Maximilian Stritzinger ${ }^{10}$, Gastón Folatelli ${ }^{11}$, Claudio Anguita ${ }^{2,27}$, \\ Gaspar Galaz $^{12}$, Elisabeth M. Green ${ }^{13}$, Chris Impey ${ }^{13}$, Yong-Cheol Kim ${ }^{14}$, Sofia Kirhakos ${ }^{15,16}$, Mathew A. Malkan ${ }^{17}$, \\ John S. Mulchaey ${ }^{8}$, Andrew C. Phillips ${ }^{18}$, Alessandro Pizzella ${ }^{19}$, Charles F. Prosser ${ }^{20,27}$, Brian P. Schmidt ${ }^{21,22}$, \\ Robert A. Schommer ${ }^{15,27}$, William Sherry ${ }^{23}$, Louis-Gregory Strolger ${ }^{24}$, Lisa A. Wells ${ }^{25}$, AND Gerard M. Williger ${ }^{26}$ \\ ${ }^{1}$ Millennium Institute of Astrophysics, Universidad de Chile, Chile; Igalbany@das.uchile.cl \\ ${ }^{2}$ Departamento de Astronomía, Universidad de Chile, Camino El Observatorio 1515, Las Condes, Santiago, Chile \\ ${ }^{3}$ Carnegie Observatories, Las Campanas Observatory, Casilla 60, La Serena, Chile \\ ${ }^{4}$ Department of Physics and Astronomy, Texas A\&M University, College Station, TX 77843, USA \\ ${ }^{5}$ The George P. and Cynthia Woods Mitchell Institute for Fundamental Physics and Astronomy, College Station, TX 77845, USA \\ ${ }^{6}$ George P. and Cynthia Woods Mitchell Institute for Fundamental Physics \& Astronomy, Texas A. \& M. University, \\ Department of Physics \& Astronomy, 4242 TAMU, College Station, TX 77843, USA \\ N. Copernicus Astronomical Center, ul. Bartycka 18, 00-716 Warszawa, Poland \\ ${ }^{8}$ The Observatories of the Carnegie Institution for Science, 813 Santa Barbara Street, Pasadena, CA 91101, USA \\ ${ }^{9}$ European Southern Observatory, Alonso de Cordova 3107, Vitacura, Casilla 19001, Santiago, Chile \\ ${ }_{11}^{10}$ Department of Physics and Astronomy, Aarhus University, Denmark \\ ${ }^{11}$ Instituto de Astrofísica de La Plata (IALP, CONICET), Argentina \\ ${ }^{12}$ Instituto de Astrofísica, Pontificia Universidad Católica de Chile, Chile \\ ${ }^{13}$ Steward Observatory, University of Arizona, 933 N. Cherry Avenue, Tucson, AZ 85721-0065, USA \\ ${ }^{14}$ Astronomy Department, Yonsei University, Seoul, Korea \\ ${ }^{15}$ Cerro Tololo Inter-American Observatory, National Optical Astronomy Observatories, Casilla 603, La Serena, Chile \\ ${ }_{16}$ Instituto de Pesquisas Espaciais, INPE, Caixa Postal 515, 12201 São José dos Campos, SP, Brazil \\ ${ }^{17}$ Astronomy Division, Department of Physics \& Astronomy, UCLA, LA, CA 90095-1547, USA \\ ${ }^{18}$ University of California Observatories, USA \\ 19 Dipartimento di Fisica e Astronomia "G. Galilei," Università di Padova, vicolo dell'Osservatorio 3, I-35122 Padova, Italy \\ ${ }^{20}$ National Optical Astronomy Observatories, 950 North Cherry Avenue, P.O. Box 26732, Tucson, AZ 85726, USA \\ ${ }^{21}$ Research School of Astronomy and Astrophysics, The Australian National University, Canberra, ACT 2611, Australia \\ ${ }^{22}$ ARC Centre of Excellence for All-sky Astrophysics (CAASTRO), Australia \\ ${ }^{23}$ Eureka Scientific, Inc., 2452 Delmer Street Suite 100, Oakland, CA 94602-3017, USA \\ ${ }^{24}$ Space Telescope Science Institute, Science Mission Office, 3700 San Martin Drive, Baltimore, MD 21218, USA \\ ${ }^{25}$ Canada-France-Hawaii Telescope Corp., 64-1238 Mamalahoa Highway, Kamuela, HI, 96743, USA \\ ${ }^{26}$ Department of Physics \& Astronomy, Univ. Louisville, Louisville, KY 40292, USA \\ Received 2015 September 1; accepted 2015 November 25; published 2016 January 27
}

\begin{abstract}
We present a compilation of UBVRIz light curves of 51 type II supernovae discovered during the course of four different surveys during 1986-2003: the Cerro Tololo Supernova Survey, the Calán/Tololo Supernova Program (C\&T), the Supernova Optical and Infrared Survey (SOIRS), and the Carnegie Type II Supernova Survey (CATS). The photometry is based on template-subtracted images to eliminate any potential host galaxy light contamination, and calibrated from foreground stars. This work presents these photometric data, studies the color evolution using different bands, and explores the relation between the magnitude at maximum brightness and the brightness decline parameter $(s)$ from maximum light through the end of the recombination phase. This parameter is found to be shallower for redder bands and appears to have the best correlation in the $B$ band. In addition, it also correlates with the plateau duration, being shorter (longer) for larger (smaller) $s$ values.
\end{abstract}

Key words: supernovae: general - surveys - techniques: photometric

Supporting material: machine-readable tables

\section{INTRODUCTION}

It is widely accepted that stars born with masses higher than $\sim 8 M_{\odot}$ explode as core-collapse supernovae (CCSN) after some tens of millions of years of evolution. At the end of their lives stars born with $\sim 8-9 M_{\odot}$ end up with an oxygen-neon-magnesium core while higher mass stars end up forming an iron core. In both cases the core grows up to reach the Chandrasekhar mass near $1.4 M_{\odot}$, and at this point the electron degeneracy pressure becomes insufficient to

\footnotetext{
${ }^{27}$ Deceased.
}

balance gravity and the core is bound to gravitational collapse. Collapse is stimulated by partial photodisintegration of Fe-group nuclei into alpha particles, and by electron capture on protons emitting neutrinos. As a result there is a decrease of the electron density, and hence the pressure at the center of the star is reduced, accelerating the collapse. This sequence of events is followed by core bounce and subsequently the ejection of the star's envelope, presumably due to energy deposited by neutrinos created in the proto-neutron core (see Ivanov \& Shulman 1990; 
Burrows 2000; Janka et al. 2007; Janka 2012 for reviews about the explosion mechanisms).

Early-time spectra of CCSNe show great diversity (Filippenko 1997). While the Type II SNe (hereafter SNe II) group consists of spectra dominated by prevalent Balmer spectral features, the Type I class is characterized by the lack of conspicuous Balmer features. The Type I class is further subdivided according to the presence of $\mathrm{He}$ in the spectrum (SNe Ib) or no He lines (SNe Ic).

The spectral differences among CCSNe are thought to be due to the relative ability of $\mathrm{SNe}$ progenitors to retain their outermost envelopes of unprocessed Hydrogen $(\mathrm{H})$ or Helium (He). In this scenario, SNe II events, which have the least massive progenitors, are those able to retain a significant fraction of their outer layers prior to explosion. On the other hand, $\mathrm{SNe} \mathrm{Ib} / \mathrm{c}$ most likely originate from massive stars that lose their $\mathrm{H}$ envelope through stellar winds (Woosley et al. 1993), mass transfer to a companion star (Nomoto et al. 1995), enhanced mixing (Frey et al. 2013), or through a combination of these processes. Supporting evidence for this scenario is available from the detection of SNe progenitors in nearby galaxies (Smartt 2015) and statistical analysis of the proximity of CCSNe to star-forming regions in their host galaxies (Anderson et al. 2012; Galbany et al. 2014).

Historically, SNe II have been sub-classified according to their photometric properties. The majority show a phase of $\sim 80$ days with a "plateau" of nearly constant luminosity (hence, historically they are referred to as SNe IIP), while a smaller fraction of "linear" SNe II show a steep initial decline (SNe IIL). Recent studies have questioned this subdivision and argue that nature provides a continuous sequence of objects, ranging from pure "slow decliners" to "fast decliners" (Anderson et al. 2014; Sanders et al. 2015). An even smaller fraction of SNe II undergo interaction of their vastly expanding ejecta with circumstellar material, which can manifest as strong narrow $\mathrm{H}$ emission lines in the spectrum, and lead to significant photometric diversity (SNe IIn, Schlegel 1990; Taddia et al. 2013).

$\mathrm{SNe}$ are not only important in the chemical enrichment of the universe and the shaping of galaxies, but also serve as accurate cosmological distance indicators. Over the past 25 years our group has been systematically studying and collecting photometric and spectroscopic data of all SN types over the course of the following surveys: (1) the Cerro Tololo Supernova Survey led by M.M.P. and N.B.S between 1986 and 1996, (2) the Calán/Tololo Supernova Program (C\&T) led by M.H., J.M., M.M.P, and N.B.S between 1989 and 1993 (Hamuy et al. 1993), (3) the Supernova Optical and Infrared Survey (SOIRS) led by M.H. between 1999 and 2000 (Hamuy 2001), and (4) the Carnegie Type II Supernova Survey (CATS) led by M.H., M.M.P, and N.B.S. between 2002 and 2003.

The purpose of this paper is to report photometric observations of $51 \mathrm{SNe}$ II obtained by these four surveys (excluding SN 1987A's data that were published in great detail by Hamuy \& Suntzeff 1990 and Phillips et al. 1990, and SNe 1990E's photometry which was presented in Schmidt et al. 1993), in order to make this data set available to the community. This data set will undoubtedly contribute to an expanded understanding of SNe II and improved methods for obtaining precise distances. Near-infrared photometry for the current sample will be presented in a separate paper. These data have been used previously for the study of specific objects
(Schmidt et al. 1994b; Zampieri et al. 2003; Hamuy et al. 2009; Krisciunas et al. 2009; Mazzali et al. 2009; Bersten et al. 2011; Takáts et al. 2015; Kleiser et al. 2011). The subsample of SNe IIP has been used for the determination of distances using the "Expanding Photosphere Method" (Schmidt et al. 1994a; Hamuy et al. 2001; Jones et al. 2009) and the "Standardized Candle Method" (Hamuy \& Pinto 2002; Nugent et al. 2006; Poznanski et al. 2009; Hamuy 2004; Olivares et al. 2010; Rodríguez et al. 2014), and for the determination of bolometric corrections (Bersten \& Hamuy 2009). Other studies that have relied on some of the objects in this sample also include: (1) Hamuy (2003a) examined the observed and physical properties of SNe II using both photometry and spectroscopy of a selection of $24 \mathrm{SNe}$ II; (2) Anderson et al. (2014) performed a characterization of the $V$-band light curves of an expanded sample of SNe II; and (3) Gutiérrez et al. (2014) have correlated those properties with the $\mathrm{H} \alpha$ feature of their spectra.

This paper is organized as follows. Section 2 summarizes our optical observations and describes the data reduction procedures; Section 3 shows an analysis of the photometric properties of the SNe II light curves, including colors, absolute magnitudes, and the brightness decline parameter $s$. Finally, a summary and the final conclusions are presented in Section 4.

\section{OBSERVATIONS}

A list of the SNe II used in this study is presented in Table 1. The table includes the following information: the SN designation and its host-galaxy names; the host-galaxy type; the SN equatorial coordinates; the heliocentric redshift of the host galaxy; the Galactic extinction, $E(B-V)_{M W}$, from the Schlafly \& Finkbeiner (2011) dust maps; the distance modulus (see Section 3.3); and the survey under which the SNe was observed. Besides the objects discovered over the course of the C\&T and SOIRS programs using photographic plates (with the Cerro Tololo Curtis-Schmidt Camera and the Cerro El Roble Maksutov Camera of the University of Chile), we also include in the list of follow-up target SNe discovered by others and reported to the IAU Circulars. Discovery and classification references for the $51 \mathrm{SNe}$ II are listed in Table 2. They are all nearby objects $(z \lesssim 0.08$, see Figure 1$)$, selected for our followup based on their relatively high apparent brightness and convenient location in the sky (decl. $\lesssim 25^{\circ}$ north).

As soon as we were notified of a discovery, and whenever we had telescope time allocated to us, detailed follow-up observations were initiated using various telescopes located at the Cerro Tololo Inter-American Observatory (CTIO), the University of Arizona's Steward Observatory, the Las Campanas Observatory of the Carnegie Institution of Science, and the European Southern Observatory (ESO) at La Silla and Paranal.

\subsection{Photometry}

The first object in our list is SN 1986L and it is the only SN observed with photoelectric techniques (by M.M.P and S.K., using the CTIO 0.9 m equipped with a photometer and $B$ and $V$ filters). The remaining SNe were observed using a variety of telescopes equipped with $\mathrm{CCD}$ detectors and $U B V(R I)_{K C} z$ filters, as indicated in Table 3. The observational techniques employed by the C\&T project are presented by Hamuy et al. (1993), and the photometric reductions are in Hamuy et al. (1996). The observations and data reductions during the SOIRS 
Table 1

Type II Supernovae

\begin{tabular}{|c|c|c|c|c|c|c|c|c|}
\hline SN Name & Host Galaxy & Host Type $^{\mathrm{a}}$ & R.A.(J2000) & decl.(J2000) & $z_{\text {helio }}{ }^{b}$ & $E(B-V)$ & DM & Survey $^{\mathrm{c}}$ \\
\hline 1986L & NGC 1559 & $\mathrm{SB}(\mathrm{s}) \mathrm{cd}$ & 041731.2 & -624707 & 0.00435 & 0.030 & $30.72(0.34)^{\mathrm{d}}$ & 1 \\
\hline 1991al & 2MASX J19422191-5506275 & $\mathrm{S}$ & 194224.0 & -550623 & $0.01525^{*}$ & 0.051 & $34.12(0.14)$ & 2 \\
\hline $1992 \mathrm{~T}$ & 2MASX J13425875-3153105 & SB & 134301.5 & -315336 & $0.03898^{*}$ & 0.048 & $36.30(0.05)$ & 2 \\
\hline $1992 U$ & ESO 074-G 004 & $\mathrm{SBc}$ & 204046.5 & -704133 & $0.01086^{*}$ & 0.051 & $33.39(0.06)$ & 2 \\
\hline 1992ad & NGC 4411B & $\mathrm{SAB}(\mathrm{s}) \mathrm{cd}$ & 122649.6 & +085239 & 0.00424 & 0.030 & $31.13(0.80)^{\mathrm{d}}$ & 2 \\
\hline 1992af & ESO 340-G 038 & $\mathrm{Sc}$ & 203040.2 & -421835 & 0.01847 & 0.052 & $34.52(0.01)$ & 2 \\
\hline 1992am & MCG-01-04-039 & $\mathrm{S}$ & 012502.7 & -043901 & $0.04799^{*}$ & 0.049 & $36.66(0.05)$ & 2 \\
\hline 1992ba & NGC 2082 & $\mathrm{SB}(\mathrm{r}) \mathrm{b}$ & 054147.1 & -641801 & 0.00395 & 0.058 & $30.41(0.80)^{\mathrm{d}}$ & 2 \\
\hline 1993A & 2MASX J07391822-6203095 & $\ldots$ & 073917.3 & -620314 & $0.02930^{*}$ & 0.173 & $35.65(0.01)$ & 2 \\
\hline $1993 K$ & NGC 2223 & $\mathrm{SAB}(\mathrm{r}) \mathrm{b}$ & 062437.8 & -224951 & 0.00908 & 0.064 & $33.15(0.40)^{\mathrm{d}}$ & 2 \\
\hline $1993 \mathrm{~S}$ & 2MASX J22522390-4018432 & S & 225223.4 & -401837 & 0.03301 & 0.016 & $35.82(0.03)$ & 2 \\
\hline $1999 \mathrm{br}$ & NGC 4900 & $\mathrm{SB}(\mathrm{rs}) \mathrm{c}$ & 130041.8 & +022946 & 0.00320 & 0.024 & $31.19(0.40)^{\mathrm{d}}$ & 3 \\
\hline 1999ca & NGC 3120 & $\mathrm{SAB}(\mathrm{s}) \mathrm{bc} ?$ & 100522.9 & -341241 & 0.00931 & 0.109 & $33.32(0.02)$ & 3 \\
\hline $1999 \mathrm{cr}$ & ESO 576-G 034 & $\mathrm{~S} / \mathrm{Irr}$ & 132018.3 & -200850 & $0.02023^{*}$ & 0.098 & $34.90(0.01)$ & 3 \\
\hline 1999eg & IC 1861 & $\mathrm{SA} 0 \wedge 0$ & 025308.4 & +252924 & 0.02236 & 0.117 & $34.94(0.01)$ & 3 \\
\hline $1999 \mathrm{em}$ & NGC 1637 & $\mathrm{SAB}(\mathrm{rs}) \mathrm{c}$ & 044127.1 & -025146 & 0.00239 & 0.040 & $30.56(0.69)^{d}$ & 3 \\
\hline $2000 \mathrm{cb}^{\mathrm{e}}$ & IC 1158 & $\mathrm{SAB}(\mathrm{r}) \mathrm{c} ?$ & 160132.1 & +014223 & 0.00643 & 0.112 & $32.51(0.59)^{\mathrm{d}}$ & 3 \\
\hline 2002ew & SDSS J205430.45-000820.9 & $\ldots$ & 205430.5 & -000826 & 0.02994 & 0.102 & $35.58(0.06)$ & 4 \\
\hline $2002 \mathrm{fa}$ & GALEXASC J205221.54+020843.8 & $\ldots$ & 205221.8 & +020842 & 0.06000 & 0.088 & $37.18(0.08)$ & 4 \\
\hline 200210 & MCG $+00-03-054$ & $\mathrm{Sbc}$ & 010116.8 & -010552 & 0.05140 & 0.036 & $36.82(0.03)$ & 4 \\
\hline $2002 \mathrm{gd}$ & NGC 7537 & SAbc? & 231437.0 & +043006 & 0.00892 & 0.067 & $32.88(0.26)^{\mathrm{d}}$ & 4 \\
\hline $2002 \mathrm{gw}$ & NGC 0922 & $\mathrm{SB}(\mathrm{s}) \mathrm{cd}$ & 022503.0 & -244751 & 0.01028 & 0.020 & $33.42(0.37)^{\mathrm{f}}$ & 4 \\
\hline $2002 \mathrm{hj}$ & NPM1G +04.0097 & $\ldots$ & 025809.3 & +044104 & 0.02360 & 0.115 & $35.07(0.03)$ & 4 \\
\hline $2002 \mathrm{hx}$ & 2MASX J08273975-1446551 & $\mathrm{SB}(\mathrm{r}) \mathrm{b} ?$ & 082739.4 & -144716 & 0.03100 & 0.054 & $35.80(0.02)$ & 4 \\
\hline 2002ig & SDSS J013637.22+005524.9 & $\ldots$ & 013636.7 & +055526 & 0.07700 & 0.034 & $37.75(0.15)$ & 4 \\
\hline $2003 B$ & NGC 1097 & $\mathrm{SB}(\mathrm{s}) \mathrm{b}$ & 024613.8 & -301345 & 0.00424 & 0.027 & $31.03(0.26)^{\mathrm{d}}$ & 4 \\
\hline 2003E & ESO 485-G 004 & $\mathrm{Sc} ?$ & 043910.9 & -241037 & $0.01490^{*}$ & 0.048 & $34.10(0.04)$ & 4 \\
\hline $2003 \mathrm{~T}$ & UGC 04864 & $\mathrm{SA}(\mathrm{r}) \mathrm{ab}$ & 091411.0 & +164448 & 0.02791 & 0.031 & $35.58(0.01)$ & 4 \\
\hline $2003 \mathrm{bg}^{\mathrm{i}}$ & ESO 420-G 009 & $\mathrm{SB}(\mathrm{s}) \mathrm{c}$ & 041059.4 & -312450 & 0.00456 & 0.022 & $33.18(0.23)^{\mathrm{h}}$ & 4 \\
\hline $2003 \mathrm{bj}$ & IC 4219 & $\mathrm{SB}(\mathrm{rs}) \mathrm{b}$ & 131829.1 & -313738 & 0.01219 & 0.065 & $33.85(0.04)$ & 4 \\
\hline 2003bl & NGC 5374 & $\mathrm{SB}(\mathrm{r}) \mathrm{bc} ?$ & 135730.6 & +060536 & $0.01459^{*}$ & 0.027 & $34.20(0.02)$ & 4 \\
\hline 2003bn & 2MASX J10023529-2110531 & $\ldots$ & 100235.5 & -211055 & 0.01277 & 0.065 & $33.97(0.02)$ & 4 \\
\hline $2003 \mathrm{ci}$ & UGC 06212 & $\mathrm{Sb}$ & 111023.8 & +044936 & 0.03037 & 0.060 & $35.78(0.06)$ & 4 \\
\hline $2003 \mathrm{cn}$ & IC 0849 & $\mathrm{SAB}(\mathrm{rs}) \mathrm{cd}$ & 130737.0 & -005650 & $0.01811^{*}$ & 0.021 & $34.68(0.03)$ & 4 \\
\hline $2003 \mathrm{cv}^{\mathrm{g}}$ & SDSS J111748.37+190905.3 & $\ldots$ & 111748.3 & +190908 & 0.02888 & 0.021 & $35.66(0.07)$ & 4 \\
\hline $2003 c x$ & NEAT J135706.53-170220.0 & $\cdots$ & 135706.4 & -170223 & 0.03700 & 0.094 & $36.18(0.03)$ & 4 \\
\hline $2003 \mathrm{dq}$ & SDSS J110445.44+152650.4 & $\ldots$ & 110445.4 & +152649 & 0.04600 & 0.019 & $36.67(0.15)$ & 4 \\
\hline $2003 \mathrm{ef}$ & NGC 4708 & $\mathrm{SA}(\mathrm{r}) \mathrm{ab}$ & 124942.2 & -110530 & $0.01480^{*}$ & 0.046 & $34.27(0.04)$ & 4 \\
\hline 2003eg & NGC 4727 & $\mathrm{SAB}(\mathrm{r}) \mathrm{bc}$ & 125058.3 & -142001 & 0.02500 & 0.053 & $35.36(0.15)$ & 4 \\
\hline 2003ej & UGC 07820 & $\mathrm{SAB}(\mathrm{s}) \mathrm{cd} ?$ & 123911.1 & +004330 & 0.01698 & 0.019 & $34.55(0.02)$ & 4 \\
\hline $2003 \mathrm{fb}$ & UGC 11522 & $\mathrm{Sbc}$ & 201150.3 & +054538 & $0.01754^{*}$ & 0.183 & $34.36(0.01)$ & 4 \\
\hline $2003 \mathrm{gd}$ & NGC 0628 & $\mathrm{SA}(\mathrm{s}) \mathrm{c}$ & 013642.6 & +154420 & 0.00219 & 0.069 & $29.93(0.40)^{\mathrm{d}}$ & 4 \\
\hline 2003hd & ESO 543-G 017 & $\mathrm{Sb}$ & 014946.3 & -215438 & 0.03950 & 0.013 & $36.23(0.01)$ & 4 \\
\hline 2003hg & NGC 7771 & $\mathrm{SB}(\mathrm{s}) \mathrm{a}$ & 235124.1 & +200638 & 0.01427 & 0.074 & $33.83(0.01)$ & 4 \\
\hline 2003hk & NGC 1085 & $\mathrm{SA}(\mathrm{s}) \mathrm{bc} ?$ & 024625.7 & +033632 & 0.02265 & 0.037 & $34.97(0.02)$ & 4 \\
\hline $2003 \mathrm{hl}$ & NGC 0772 & $\mathrm{SA}(\mathrm{s}) \mathrm{b}$ & 015921.3 & +190015 & 0.00825 & 0.073 & $32.60(0.18)^{\mathrm{d}}$ & 4 \\
\hline $2003 \mathrm{hn}$ & NGC 1448 & SAcd? & 034436.1 & -443749 & 0.00390 & 0.014 & $31.13(0.25)^{\mathrm{d}}$ & 4 \\
\hline 2003ho & ESO 235-G 058 & $\mathrm{SB}(\mathrm{rs}) \mathrm{d}$ & 210630.5 & -480730 & 0.01438 & 0.039 & $33.95(0.04)$ & 4 \\
\hline $2003 \mathrm{ib}$ & ESO 528-G 018 & $(\mathrm{R}) \mathrm{S}(\mathrm{r}) \mathrm{b}$ ? & 203331.0 & -243715 & 0.02482 & 0.048 & $35.17(0.01)$ & 4 \\
\hline 2003ip & UGC 00327 & $\mathrm{Sbc}$ & 003316.7 & +075420 & 0.01801 & 0.066 & $34.39(0.01)$ & 4 \\
\hline $2003 i q$ & NGC 0772 & $\mathrm{SA}(\mathrm{s}) \mathrm{b}$ & 015919.9 & +185942 & 0.00825 & 0.073 & $32.60(0.18)^{\mathrm{d}}$ & 4 \\
\hline
\end{tabular}

Notes.

${ }^{a}$ From NASA/IPAC Extragalactic Database (NED).

${ }^{\mathrm{b}}$ From NED except those marked with ${ }^{*}$ which have been measured here.

c (1) Cerro Tololo Supernova Survey; (2) Calán/Tololo Supernova Program (C\&T); (3) Supernova Optical and Infrared Survey (SOIRS); (4) Carnegie Type II Supernovae Survey (CATS).

${ }^{d}$ From NED using only Tully-Fisher measurements. Errors are the standard deviation of the mean.

e Peculiar 1987A-like SN.

${ }^{f}$ No Tully-Fisher measurements available. DM measured used SNe II data.

gype IIb SN.

${ }^{\mathrm{h}}$ Although its redshift is lower than 0.01, it has been measured using Tully-Fisher measurements.

i Type IIn SN.

(This table is available in its entirety in machine-readable form.) 
Table 2

Discovery and Classification References for the 51 Type II Supernovae

\begin{tabular}{|c|c|c|}
\hline SN Name & Discovery & Classification \\
\hline $1986 \mathrm{~L}$ & Evans et al. (1986) & Lloyd Evans et al. (1986) \\
\hline 1991al & Wells et al. (1991) & Bouchet et al. (1991) \\
\hline $1992 \mathrm{~T}$ & Maza et al. (1992b) & $\ldots^{\mathrm{a}}$ \\
\hline $1992 \mathrm{U}$ & Maza et al. (1992a) & Phillips (1992) \\
\hline 1992ad & McNaught et al. (1992) & McNaught et al. (1992) \\
\hline 1992af & Wells et al. (1992) & della Valle \& Bianchini (1992) \\
\hline 1992am & Phillips et al. (1992) & Phillips et al. (1992) \\
\hline 1992ba & Evans \& Phillips (1992) & Evans \& Phillips (1992) \\
\hline 1993A & Maza et al. (1993b) & Phillips (1993a) \\
\hline $1993 \mathrm{~K}$ & Williams et al. (1993) & Hamuy (1993a) \\
\hline $1993 S$ & Maza et al. (1993a) & Hamuy (1993b) \\
\hline $1999 \mathrm{br}$ & King (1999) & Garnavich et al. (1999) \\
\hline $1999 \mathrm{ca}$ & Woodings et al. (1999) & Patat et al. (1999) \\
\hline $1999 \mathrm{cr}$ & Maza et al. (1999) & Maza et al. (1999) \\
\hline 1999eg & Hurst et al. (1999) & Jha et al. (1999b) \\
\hline $1999 \mathrm{em}$ & Li (1999) & Jha et al. (1999a) \\
\hline $2000 \mathrm{cb}$ & Papenkova \& Li (2000) & Papenkova \& Li (2000) \\
\hline 2002ew & Hutchings et al. (2002) & Filippenko \& Chornock (2002) \\
\hline $2002 \mathrm{fa}$ & Wood-Vasey et al. (2002b) & Hamuy (2002b) \\
\hline 200210 & $\ldots^{\mathrm{b}}$ & \\
\hline $2002 \mathrm{gd}$ & Klotz et al. (2002) & Hamuy (2002a) \\
\hline $2002 \mathrm{gw}$ & Monard (2002) & Hamuy et al. (2002) \\
\hline 2002hj & Wood-Vasey et al. (2002a) & Chornock et al. (2002) \\
\hline $2002 \mathrm{hx}$ & Graham \& Li (2002) & Matheson et al. (2002) \\
\hline 2002ig & Miknaitis et al. (2002) & Miknaitis et al. (2002) \\
\hline 2003B & Evans \& Quirk (2003) & Kirshner \& Silverman (2003) \\
\hline 2003E & Papenkova \& Li (2003) & Hamuy (2003d) \\
\hline $2003 \mathrm{~T}$ & Poznanski et al. (2003) & Foley et al. (2003) \\
\hline 2003bg & Wood-Vasey et al. (2003c) & Hamuy et al. (2003c) \\
\hline $2003 b j$ & Chassagne (2003) & Swift et al. (2003) \\
\hline 2003bl & Swift et al. (2003) & Phillips et al. (2003) \\
\hline 2003bn & Wood-Vasey et al. (2003d) & Salvo et al. (2003b) \\
\hline $2003 \mathrm{ci}$ & Revnivtsev et al. (2003) & Salvo et al. (2003b) \\
\hline $2003 \mathrm{cn}$ & Wood-Vasey et al. (2003e) & Hamuy (2003b) \\
\hline $2003 \mathrm{cv}$ & Wood-Vasey et al. (2003b) & Hamuy et al. (2003a) \\
\hline $2003 c x$ & Wood-Vasey et al. (2003a) & Hamuy (2003c) \\
\hline $2003 d q$ & Puckett et al. (2003) & Phillips \& Hamuy (2003) \\
\hline $2003 \mathrm{ef}$ & Weisz \& Li (2003) & Ganeshalingam et al. (2003) \\
\hline 2003eg & Ganeshalingam \& Li (2003a) & Matheson et al. (2003b) \\
\hline 2003 еj & Ganeshalingam et al. (2003) & Matheson et al. (2003c) \\
\hline $2003 \mathrm{fb}$ & Papenkova et al. (2003) & Papenkova et al. (2003) \\
\hline $2003 \mathrm{gd}$ & Evans \& McNaught (2003) & Garnavich \& Bass (2003) \\
\hline 2003hd & Ganeshalingam \& Li (2003b) & Hamuy et al. (2003b) \\
\hline 2003hg & Moore \& Li (2003) & Elias-Rosa et al. (2003) \\
\hline $2003 \mathrm{hk}$ & Boles \& Li (2003) & Filippenko et al. (2003) \\
\hline 2003hl & Moore et al. (2003) & Filippenko et al. (2003) \\
\hline $2003 \mathrm{hn}$ & Evans et al. (2003) & Salvo et al. (2003a) \\
\hline 2003ho & Monard (2003) & Hamuy \& Roth (2003) \\
\hline 2003ib & Singer et al. (2003) & Morrell \& Hamuy (2003) \\
\hline 2003ip & Li et al. (2003) & Filippenko \& Foley (2003) \\
\hline $2003 i q$ & Llapasset et al. (2003) & Matheson et al. (2003a) \\
\hline
\end{tabular}

Notes.

${ }^{\text {a }}$ Not reported in the literature. Classification provided here for the first time.

b The CATS survey performed the follow up of SNe 200210, which was discovered by the SN Factory (Wood-Vasey et al. 2004) and was never reported to the International Astronomical Union (IAU) to provide an official designation.

project are explained in Hamuy (2001), whereas the techniques employed during the CATS project can be found in Hamuy et al. (2009). We also refer the reader to Hamuy et al. (2006), which describes the observational procedures of the Carnegie

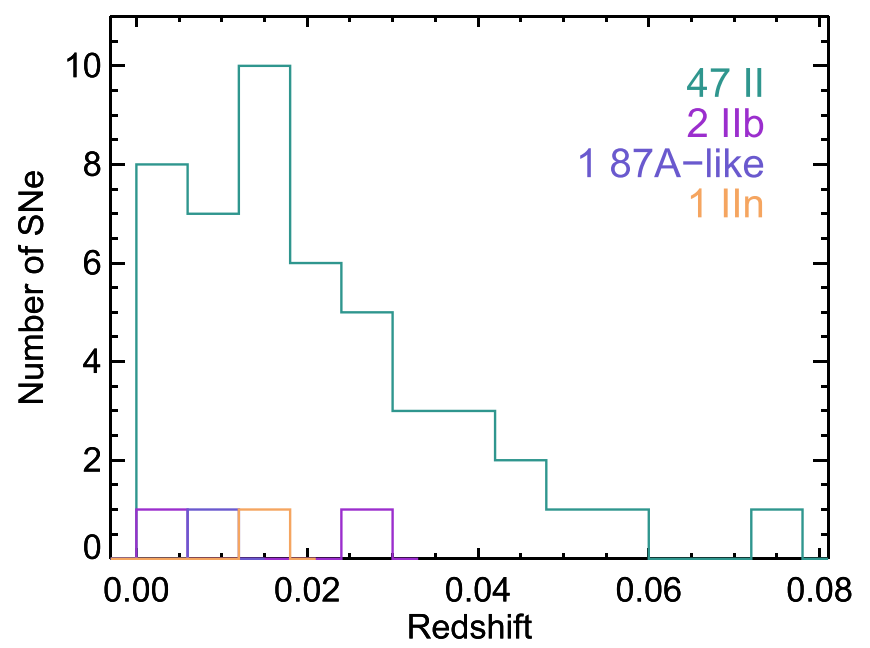

Figure 1. Redshift distribution of the $51 \mathrm{SNe}$ II presented in this work, separated into normal SN II, IIb, and peculiar events. The median value of the distribution is 0.017 , the average value is 0.021 , and the standard deviation is 0.016 .

Table 3

Telescope and Instruments Employed for Photometry

\begin{tabular}{lcc}
\hline \hline Observatory & Telescope & Instrument \\
\hline Cerro Tololo & $0.9 \mathrm{~m}$ & Photoelectric Photometer \\
Cerro Tololo & $0.9 \mathrm{~m}$ & CCD Camera \\
Cerro Tololo & $1.0 \mathrm{~m}$ & CCD Camera \\
Cerro Tololo & $1.5 \mathrm{~m}$ & CCD Camera \\
Cerro Tololo & Blanco $4 \mathrm{~m}$ & CCD Camera \\
Las Campanas & Swope $1 \mathrm{~m}$ & CCD Camera \\
Las Campanas & du Pont $2.5 \mathrm{~m}$ & CCD Camera \\
Las Campanas & Baade $6.5 \mathrm{~m}$ & CCD Camera \\
Las Campanas & Clay $6.5 \mathrm{~m}$ & CCD Camera \\
La Silla & $1.54 \mathrm{~m}$ & CCD Camera \\
La Silla & NTT $3.5 \mathrm{~m}$ & CCD Camera \\
Steward & $2.3 \mathrm{~m}$ & CCD Camera \\
Steward & $1.6 \mathrm{~m}$ & CCD Camera \\
\hline
\end{tabular}

Supernova Project (CSP), which are nearly identical to those of CATS. In fact, CATS was a precursor to the CSP initiated in 2004 with the aim to study $\mathrm{SNe}$ of all types. One main difference between CATS and the original format of the CSP is that the latter uses SDSS ugri filters in addition to Johnson $B$ and $V$ filters for optical imaging (Stritzinger et al. 2011).

All photometric reductions were performed with customized IRAF $^{28}$ scripts. In brief, the photometric reductions begin by subtracting host-galaxy template images from the $\mathrm{SNe}+$ galaxy images. The templates are high signal-to-noise images (in each filter) of the SN field obtained under good seeing conditions after the SN has faded from detection. As a result of this procedure the $\mathrm{SNe}$ generally end up lying on a smooth background, allowing us to reliably measure the SN flux with no contamination from the host-galaxy background. The next step is to compute differential photometry of the SN with respect to a local sequence of stars calibrated relative to standard star observations obtained over multiple photometric nights. For this, we measured instrumental magnitudes of the

\footnotetext{
${ }^{28}$ IRAF is distributed by the National Optical Astronomy Observatories, which are operated by the Association of Universities for Research in Astronomy, Inc., under cooperative agreement with the National Science Foundation.
} 
SN and the local sequence stars via PSF fitting when the SN was faint, or simple aperture photometry when the object was bright. The transformation of the instrumental magnitudes to the standard $U B V(R I)_{K C} z$ system assumes a linear term in magnitude, a color term, and a photometric zeropoint,

$$
X=x+C T_{X}+Z P_{X},
$$

where $X$ represents the standard system magnitude, $x$ is the instrumental magnitude, the color term $C T_{X}$ is an average measured over many photometric nights for each telescope/ $\mathrm{CCD} /$ filter combination, and the zeropoint $Z P_{X}$ is a fitting parameter determined from all the local standards. Note that there is no atmospheric extinction term because it is absorbed by the zeropoint, when doing differential photometry.

The photometric sequences for the $51 \mathrm{SNe}$ II are identified in the finding charts in Figure 2 and their magnitudes are listed in Table 4 along with the standard error of the mean (in units of mmag) and the number of nights on which each star was observed. In every case, these sequences were derived from observations of Landolt standards (see Appendix D in Hamuy et al. 2001 for the definition of the $z$ band and Stritzinger et al. 2002 for the description of the $z$-band standards). Table 5 lists the resulting UBVRIz magnitudes for the $51 \mathrm{SNe}$. The uncertainties are shown in parenthesis and the telescope is indicated for each observation. The uncertainties correspond to the photon Poisson statistics, adopting a minimum error of $0.015 \mathrm{mag}$, which is typical for a single observation of the Landolt standards with CCD detectors. In total we provide a data set with 2516 photometric points.

\subsection{Spectroscopy}

In addition to broad-band photometry, several epochs of visual-wavelength spectra were obtained for this SN set, which are used here to aid in the determination of the explosion epoch (See Section 3.1) The number of epochs per object ranges between 1 and 27, with $\sim 7$ spectra being obtained per object on average. Spectral epochs are shown in Figure 3 with short vertical brown solid lines. All optical spectral sequences, with their reduction and analysis, will be presented in a future publication (C. Gutiérrez et al. 2016, in preparation).

\section{ANALYSIS}

Based on their spectral features and/or light curve morphologies, a handful of $\mathrm{SNe}$ in our sample have been classified as SNe IIb or SNe II peculiar. This includes: SN 2000cb which shows similar photometric behavior to SN 1987A; SN 2003bg has been classified as SN Ilb and studied by Hamuy et al. (2009); SN 2003cv is similar to 2003bg and has also been classified as SN Ilb; SN 2003bj showed signs of SN+CSM interaction in its spectra and has been classified as SN IIn. They are all plotted using different symbols and sometimes excluded in the following analysis.

\subsection{Individual Multi-band Light Curves}

Multi-band light curves showing their cadence and quality are presented in Figure 3, all referenced to their explosion epoch, which has been determined in a similar fashion as in Anderson et al. (2014). When non-detections are available the intermediate epoch between the last non-detection and the first detection is taken as an approximation of the explosion day, and its error is assumed to be half of this duration. In cases with no non-detections available or when the last non-detection is older than 20 days, the explosion epoch has been determined by matching spectral templates to our optical spectra using SNeID (Blondin \& Tonry 2007) and averaging the epoch of the best fits. For this, the spectra of $\mathrm{SNe}$ with well constrained explosion epochs from non-detections have been incorporated as new templates to SNeID (exact details will be given in $\mathrm{C}$. Gutiérrez et al. 2016, in preparation).

All photometric measurements have been corrected for Galactic extinction using dust maps from Schlafly \& Finkbeiner (2011) assuming an $R_{V}=3.1$ and a Cardelli et al. (1989) law. No correction for SN host galaxy extinction is applied to the data, and neither $S$ - nor $K$-corrections have been considered due to the similar bands used in the observations and the low redshift range of our data (Sanders et al. 2015 and T. de Jaeger et al. 2016, in preparation showed that the $K$ terms are lower than $0.2 \mathrm{mag}$ at redshifts lower than 0.1 ). In addition, the temporal scale of all light curves has been corrected for time dilation. All further analysis presented in the following sections starts with these corrections applied.

In Figure 4 we show the temporal coverage of our objects sorted by the first (left panel) and last photometric epoch (right panel), all with respect to the estimated explosion date. For the vast majority of objects, the first observation was performed within 20 days from explosion $\left(\left\langle t_{\text {first }}\right\rangle=14.7 \pm 11.1\right.$ days $)$ and on average the light curves extend through $158.2 \pm 98.7$ days, covering the whole recombination phase.

\subsection{Color Curves}

Our multi-band light curves allow for the study of the color characteristics and its temporal evolution. In Figure 5 the behavior of the $(U-B),(B-V),(B-R),(B-I),(V-R)$, $(V-I)$ and $(R-I)$ colors is presented. In the top panel, average values of the color curves binned in 30 day intervals are overploted, where the horizontal error represents the width of the bin and the vertical error represents the standard deviation for the objects in each bin. The bottom panels show each color separately.

All colors increase steadily at early times during the first few weeks due to the drop in temperature, which shifts the peak of the spectral energy distribution to redder wavelengths. This initial slope is more pronounced in colors containing bluer bands or with greater wavelength baselines, because bluer bands are more sensitive to the temperature decline and the increasingly strong line blocking affecting this initial phase (Dessart et al. 2013). In the subsequent weeks the increase is less pronounced because the temperature conditions at the photosphere remain similar due to the recombination of $\mathrm{H}$ happening during this phase (Chieffi et al. 2003). During the radioactive phase (after 100-150 days from explosion) the color curves become flatter, in part because in this phase the SN II photometric evolution, which depends on the ${ }^{56}$ Co decay, is approximately the same in all bands. At later epochs, when approaching the nebular phase $(\lesssim 200$ days $)$ all curves start to decrease, the spectrum shows weak continuum, and the emission lines start to dominate.

The range spanned by a given color index decreases in the following order: $(B-I),(B-R),(B-V),(V-I),(V-R)$, and $(R-I)$. The same sequence is also seen in the scatter for a color index at a given epoch. It has already been shown that scatter in SN II intrinsic color evolution exists, rendering it difficult to determine dust absorption for individual $\mathrm{SNe}$ 


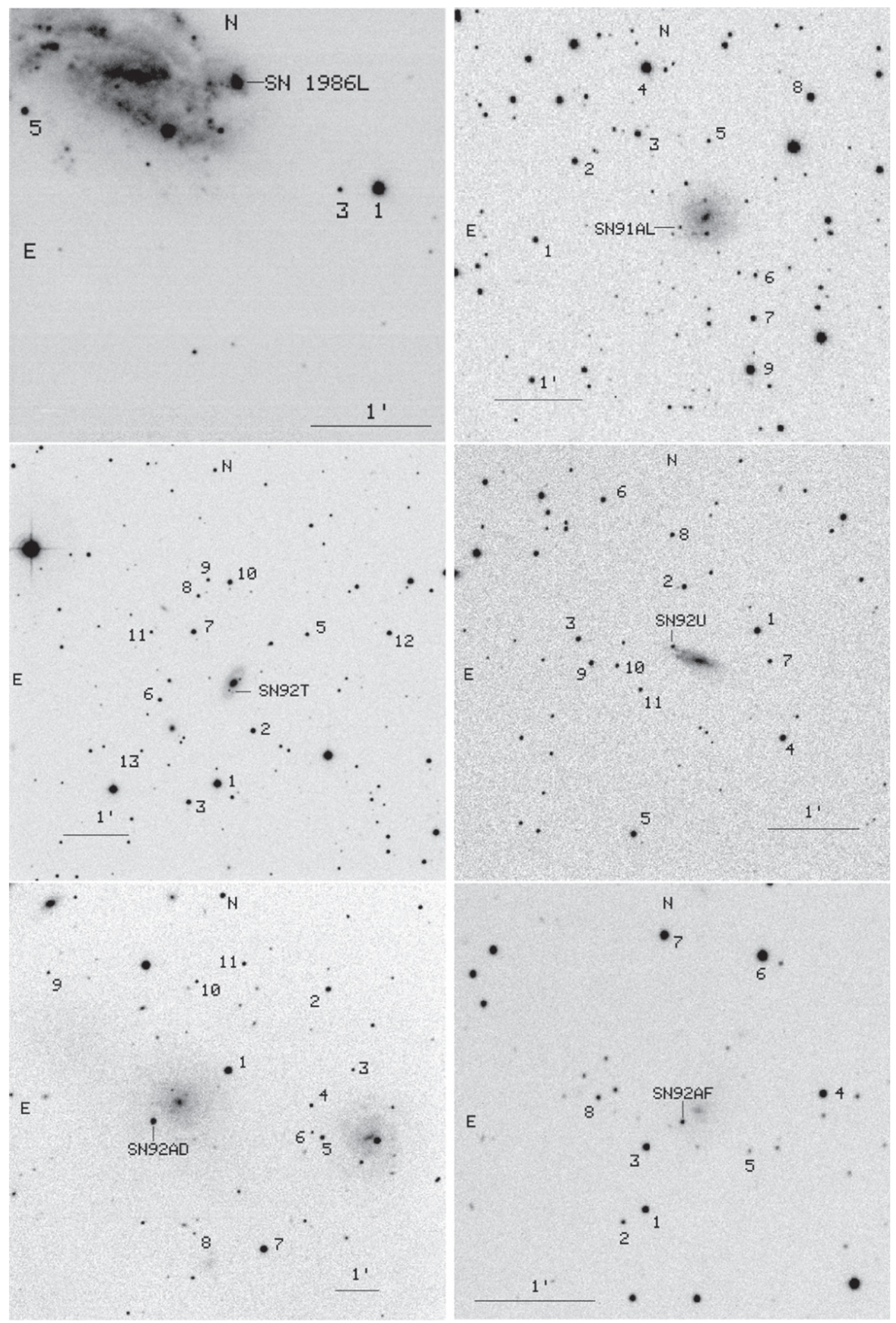

Figure 2. $V$-band images of the $51 \mathrm{SNee}$. North is up and east is to the left. Both the supernova and the comparison stars used to derive differential photometry of the SNe are labeled. The scale is shown with an horizontal line near the bottom. Here are supernovae 1986L, 1991al, 1992 T, 1992U, 1992ad, and 1992af.

(Dessart et al. 2013; Pejcha \& Prieto 2015b). Keeping this caveat in mind, and assuming that those objects with the bluest colors suffer little to no reddening, one can interpret the color excess as an indication of the amount of extinction (Schmidt et al. 1992). This assumption agrees with the fact that the color excesses decrease in the redder bands, implying lower extinction with increasing wavelength. Further analysis on host galaxy extinction is beyond the scope of this paper, and will be presented in T. de Jaeger et al. (2016, in preparation). 


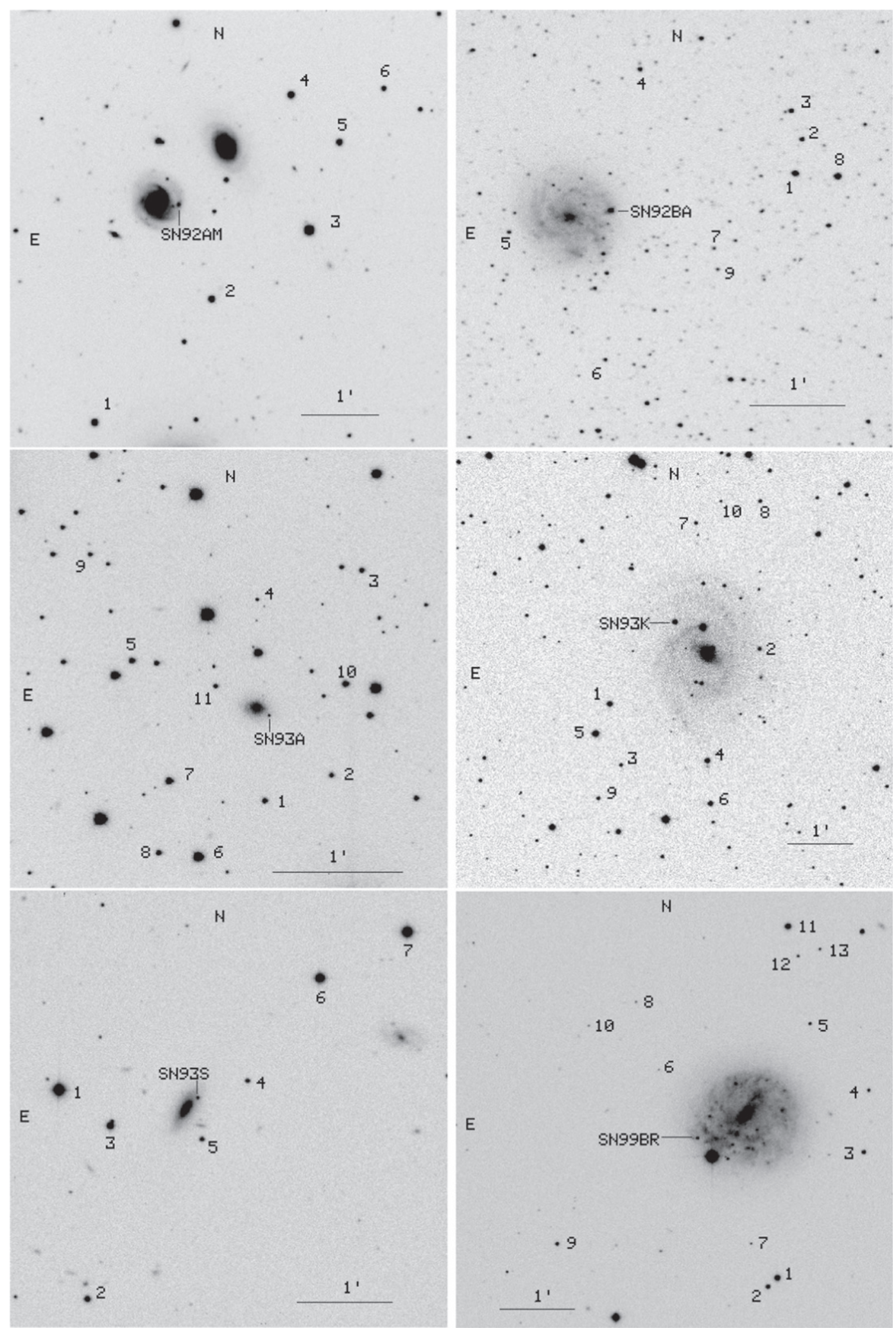

Figure 2. (Continued.)

For the few objects with $U$ and $B$ photometry available we also show in Figure 5 the $(U-B)$ color with blue empty squares. It is clearly seen how at early epochs it takes negative values, showing that right after explosion $\mathrm{SNe}$ II emit more intensely in shorter wavelengths than in the optical. In the days following the explosion SN II spectra are characterized by a blue and featureless continuum. In the following weeks, in addition to the temperature decrease, line blanketing affects the UV part of the spectrum, making the UV brightness decline steeper than the blue, and producing a rapid $(U-B)$ increase. 


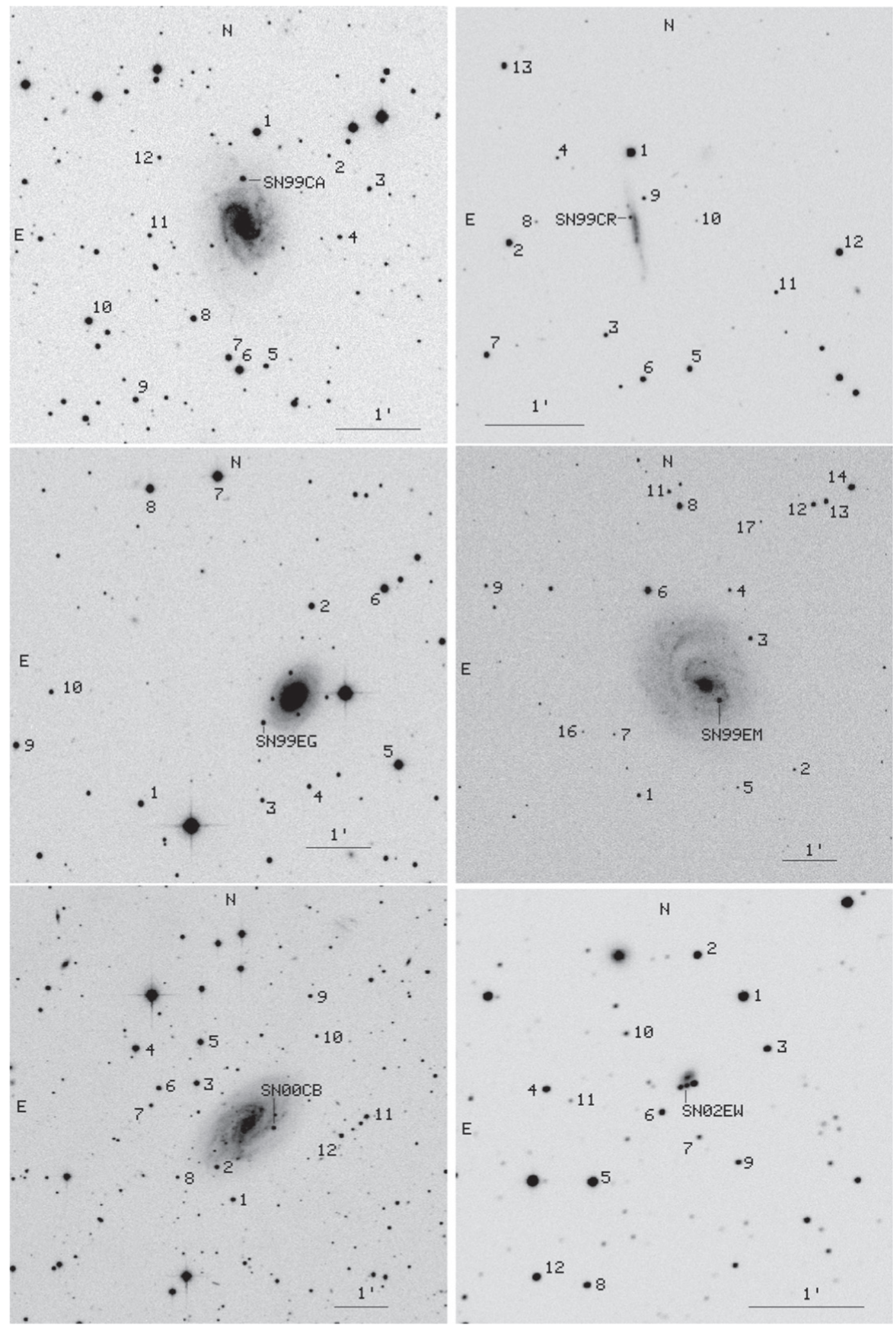

Figure 2. (Continued.)

In general all objects show similar behavior in all colors, with some special cases. We find a few objects further than $2 \sigma$ from the average color curve. The three objects with the reddest colors are SNe 2003fb, 2003hg and 2003ho. SN 2003hl is intermediate between these three and the bulk of objects. These objects probably suffer important levels of extinction. On the other hand, SN 1999em (together with SNe 1991al, $1993 \mathrm{~K}, 2002 \mathrm{ew}, 2003 \mathrm{~B}, 2003 \mathrm{bn}$, and 2003ib) is located at the bottom of the curves, which can be interpreted as having little host-galaxy reddening. Also shown in solid lines is the color evolution of the four objects that were classified as 1987A-like, SN IIb, or SN IIn. SN 2003cv is also 


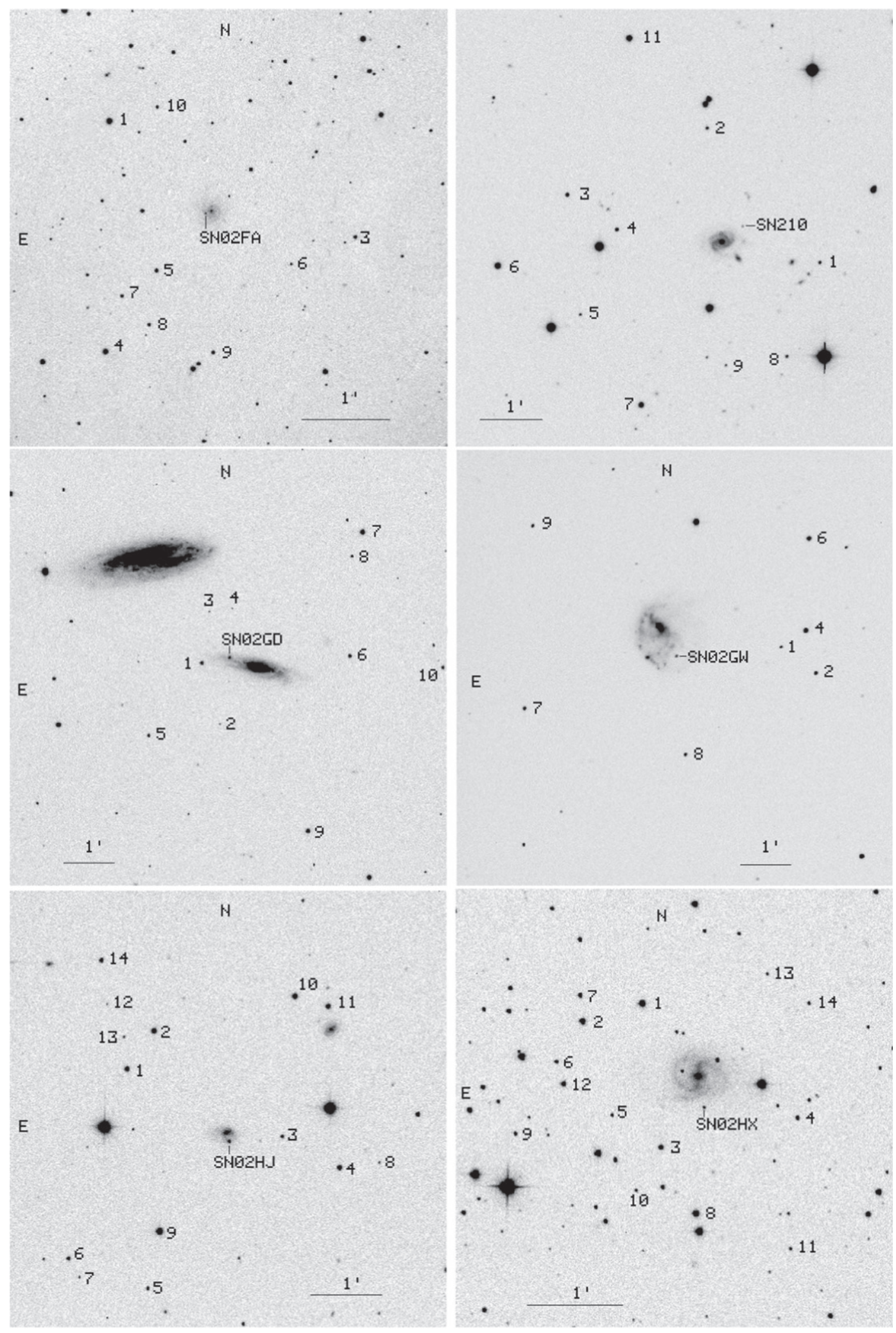

Figure 2. (Continued.)

located at the redder end in the color curve evolution at early epochs, while SN 2003bg (also a SNe IIb) follows the average evolution, but is bluer than all other SNe II 50 days after explosion.
Although we did not differentiate our sample into SN IIP and SN IIL subtypes, we do not see two distinct color behaviors in any of the color curves, therefore confirming that $\mathrm{SNe}$ II form a continuous class. 


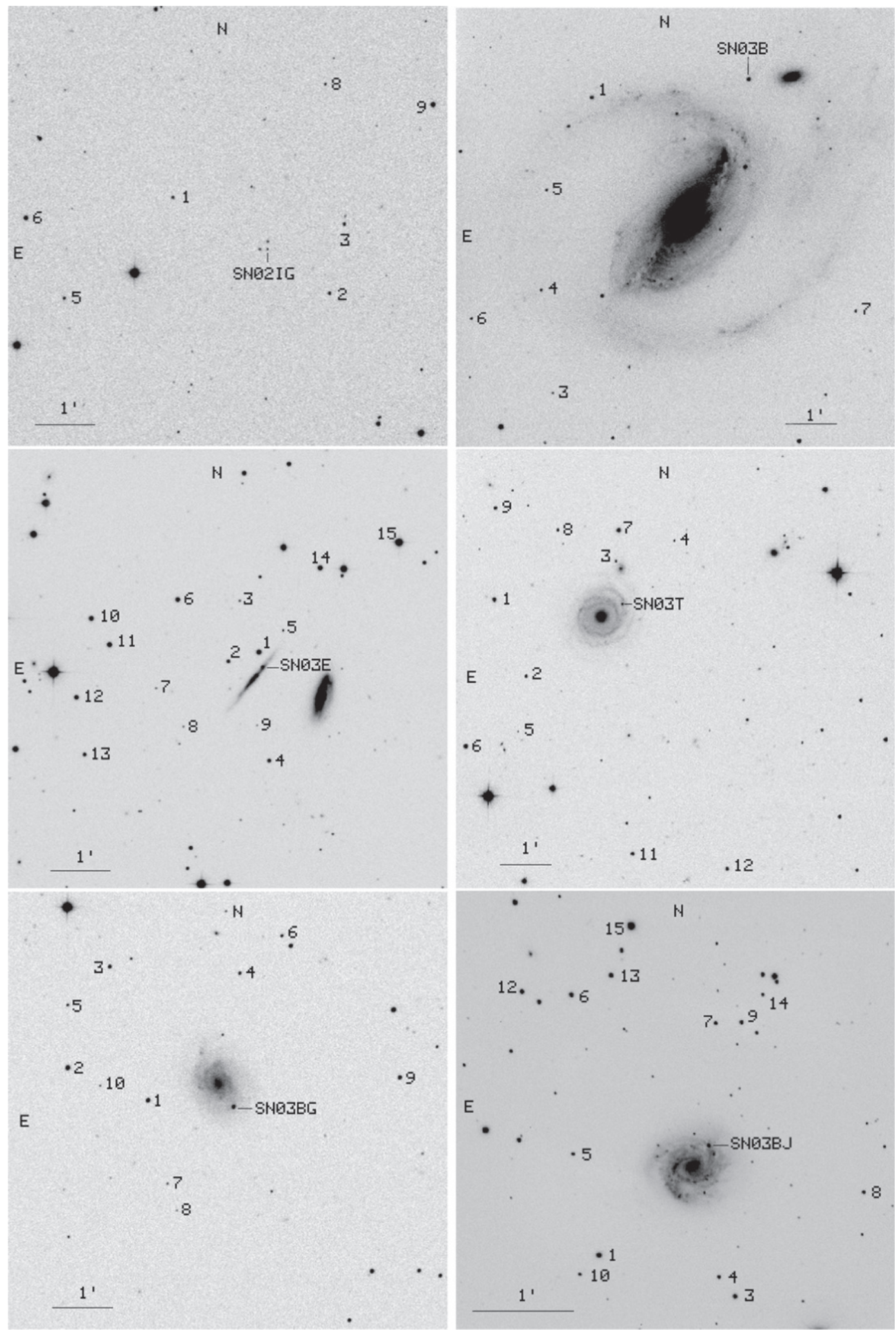

Figure 2. (Continued.)

\subsection{Absolute Magnitudes}

We calculated absolute magnitudes of all our photometric measurements to study the global behavior of SNe II light curves in different bands. For that, a measurement of the distance to the $\mathrm{SNe}$ is needed. Distances to $\mathrm{SNe}$ host galaxies with cosmic microwave background-corrected recession velocities lower than $3000 \mathrm{~km} \mathrm{~s}^{-1}$ are collected from NED and averaged, using only distances based on the Tully-Fisher and Cepheids methods. For SNe with host galaxy velocities higher than $3000 \mathrm{~km} \mathrm{~s}^{-1}$ the distance is measured using the luminosity distance expression, 


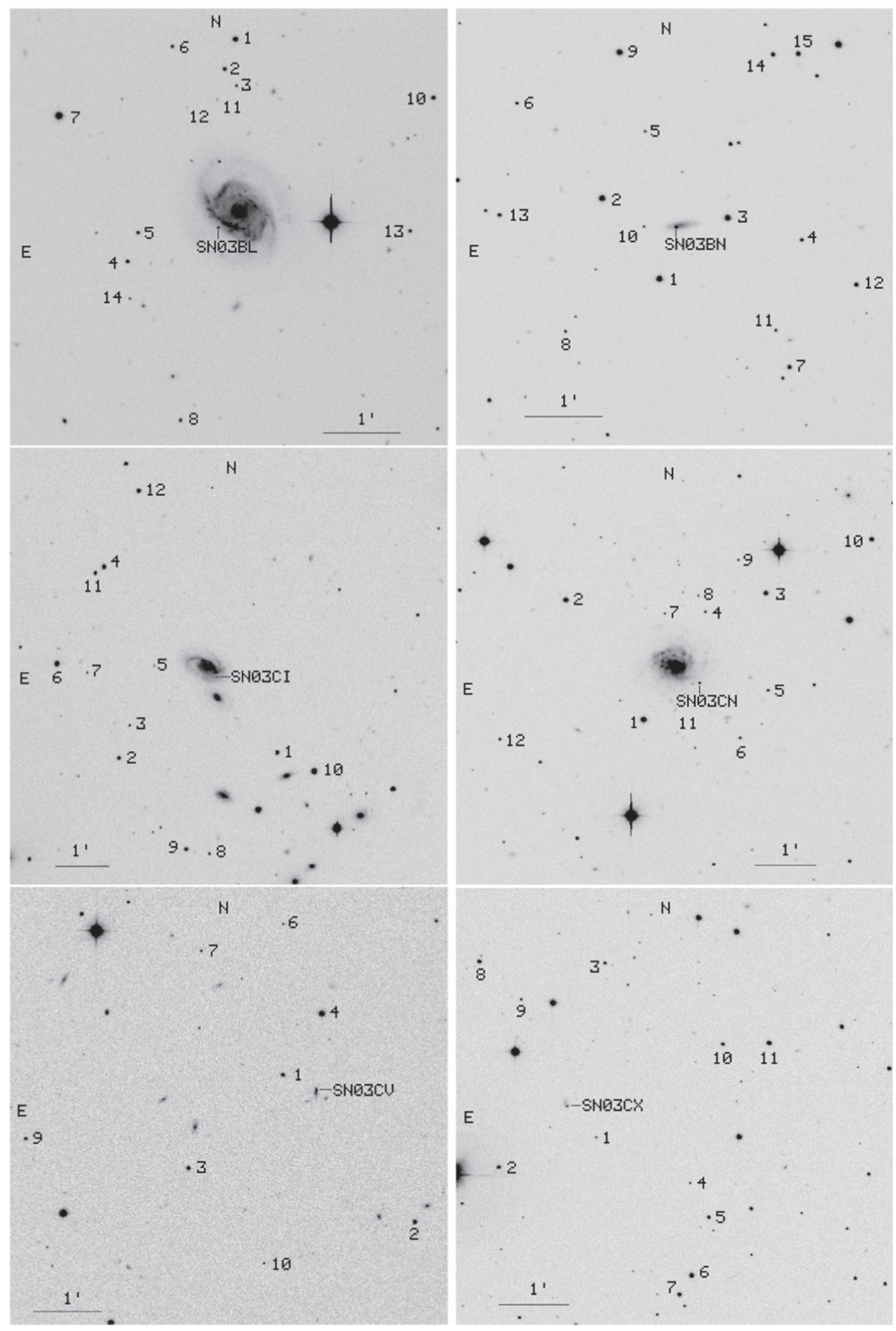

Figure 2. (Continued.)

assuming a Hubble constant, $H_{0}$, of $68 \mathrm{~km} \mathrm{~s}^{-1} \mathrm{Mpc}^{-1}$ and cosmological density parameters $\Omega_{M}=0.30$ and $\Omega_{\Lambda}=0.70$ (Planck Collaboration et al. 2015). Distance errors are added to the photometric errors in the absolute magnitude error budget. Figure 6 shows all the available MW extinction-corrected absolute magnitude light curves for each of the six bands presented here in separated panels, smoothed using a third order spline polynomial. All panels show wide ranges in both absolute magnitudes and light-curve morphologies of the $\mathrm{SNe}$ presented here. 


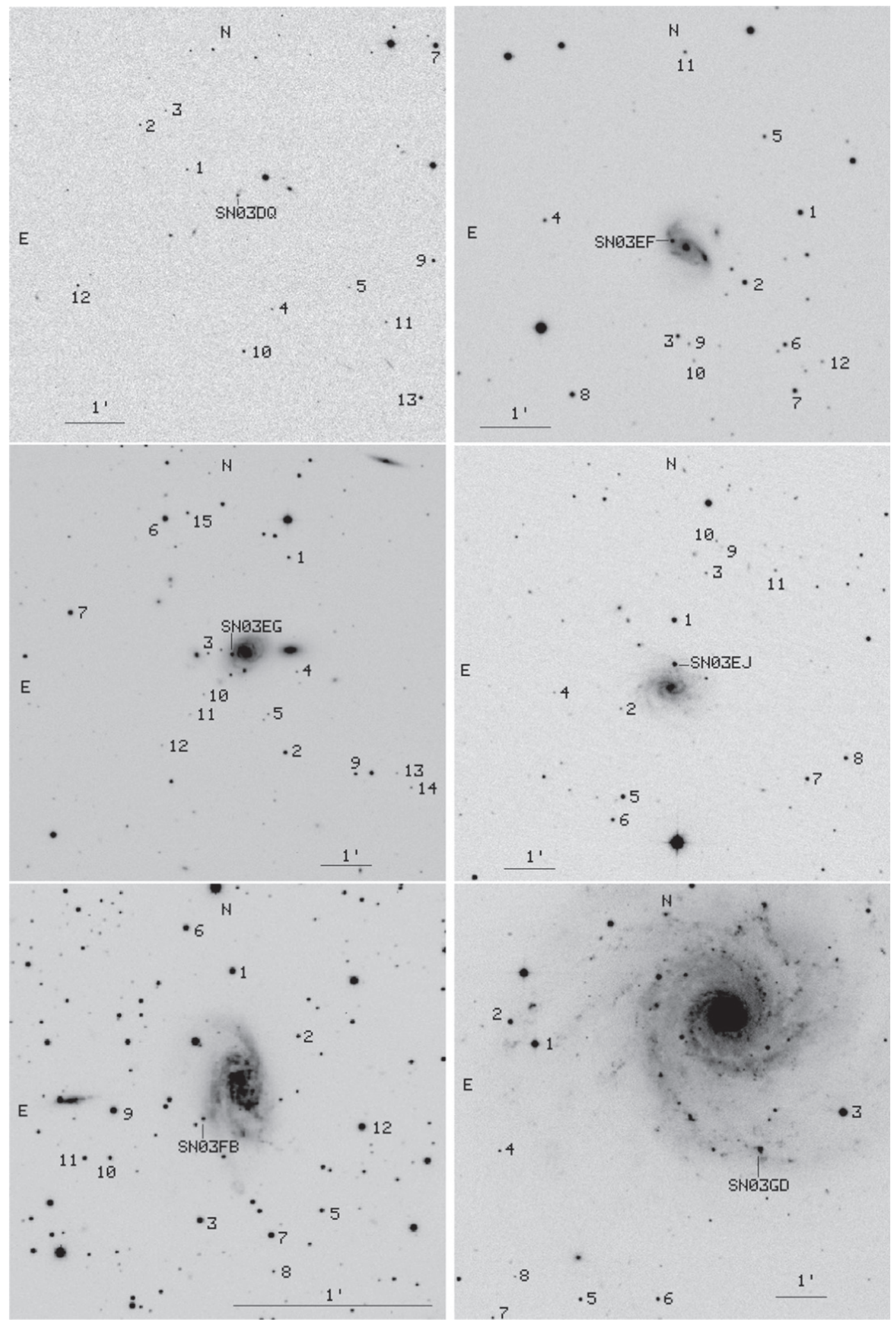

Figure 2. (Continued.)

Absolute magnitude at maximum brightness in all light curves were measured by fitting a second order polynomial to the early epochs when: (i) at least four points were available, and (ii) the second measurement was brighter than the first (indicating that the first was still part of the rise). Otherwise the first photometric point was considered as the maximum brightness of the SN.

The average MW extinction-corrected peak absolute magnitudes of all SNe in our sample (excluding 87A-like, IIb, and IIn) are the following: $\left\langle U_{\max }\right\rangle=-16.06 \mathrm{mag}(\sigma=1.74,6$ 


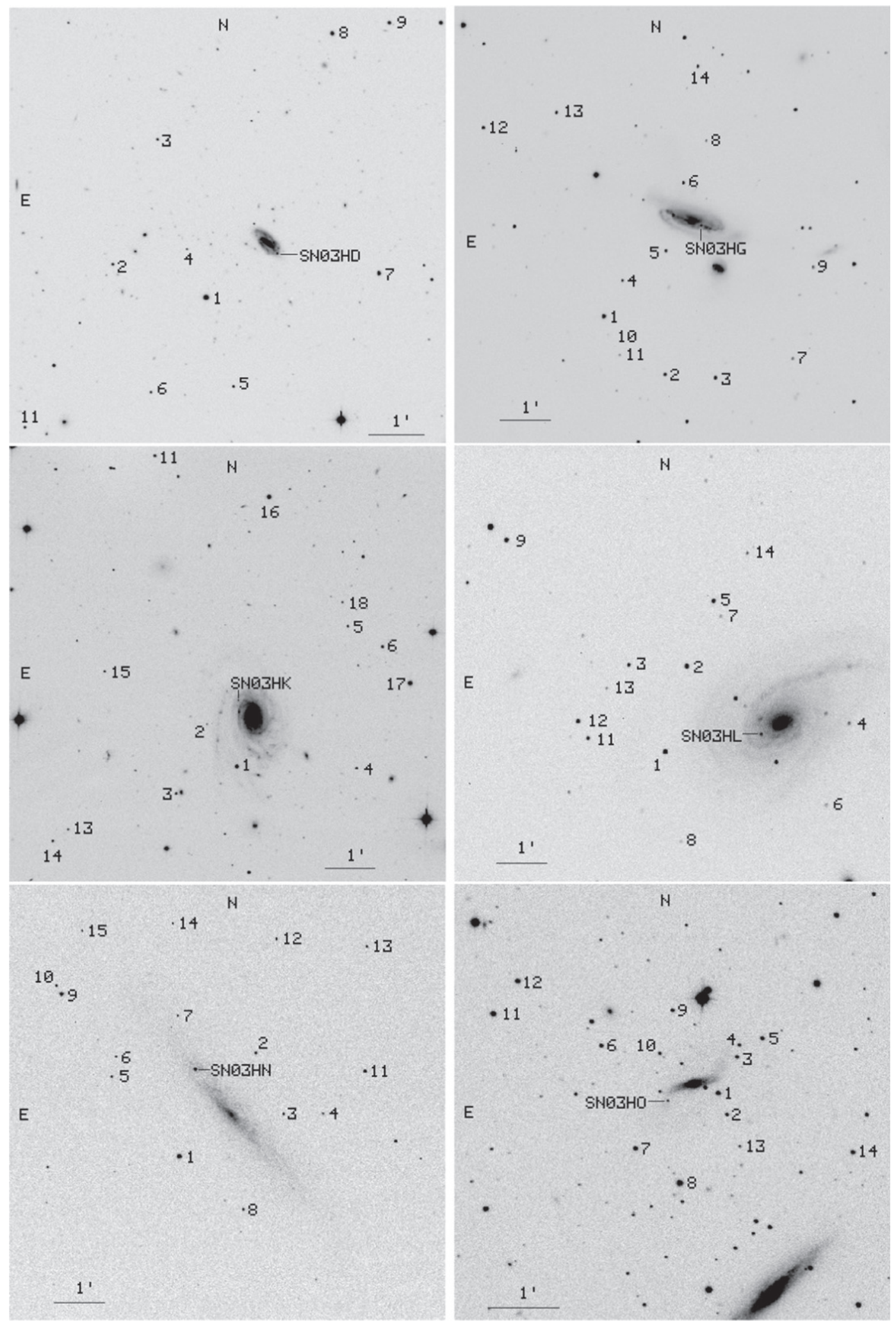

Figure 2. (Continued.)

$\mathrm{SNe}) ;\left\langle B_{\max }\right\rangle=-16.43 \mathrm{mag}(\sigma=1.19,47 \mathrm{SNe}) ;\left\langle V_{\max }\right\rangle=$ $-16.89 \mathrm{mag} \quad(\sigma=0.98, \quad 45 \mathrm{SNe}) ;\left\langle R_{\max }\right\rangle=-16.96 \mathrm{mag}$ $(\sigma=1.03,24 \mathrm{SNe}) ;\left\langle I_{\max }\right\rangle=-17.38 \mathrm{mag}(\sigma=0.95,46$ $\mathrm{SNe})\left(\left\langle z_{\max }\right\rangle\right.$ is not reported since there are not enough data to do statistics). The average absolute magnitude increases by
1.3 mag from $U$ to $I$. We added a horizontal line and a strip in Figure 6, representing the average absolute peak magnitude and its $1 \sigma$ scatter, respectively. Light curves in our sample fill the strip at peak epochs, showing a continuum of peak absolute magnitudes. Similarly to Faran et al. (2014a, 2014b), our 


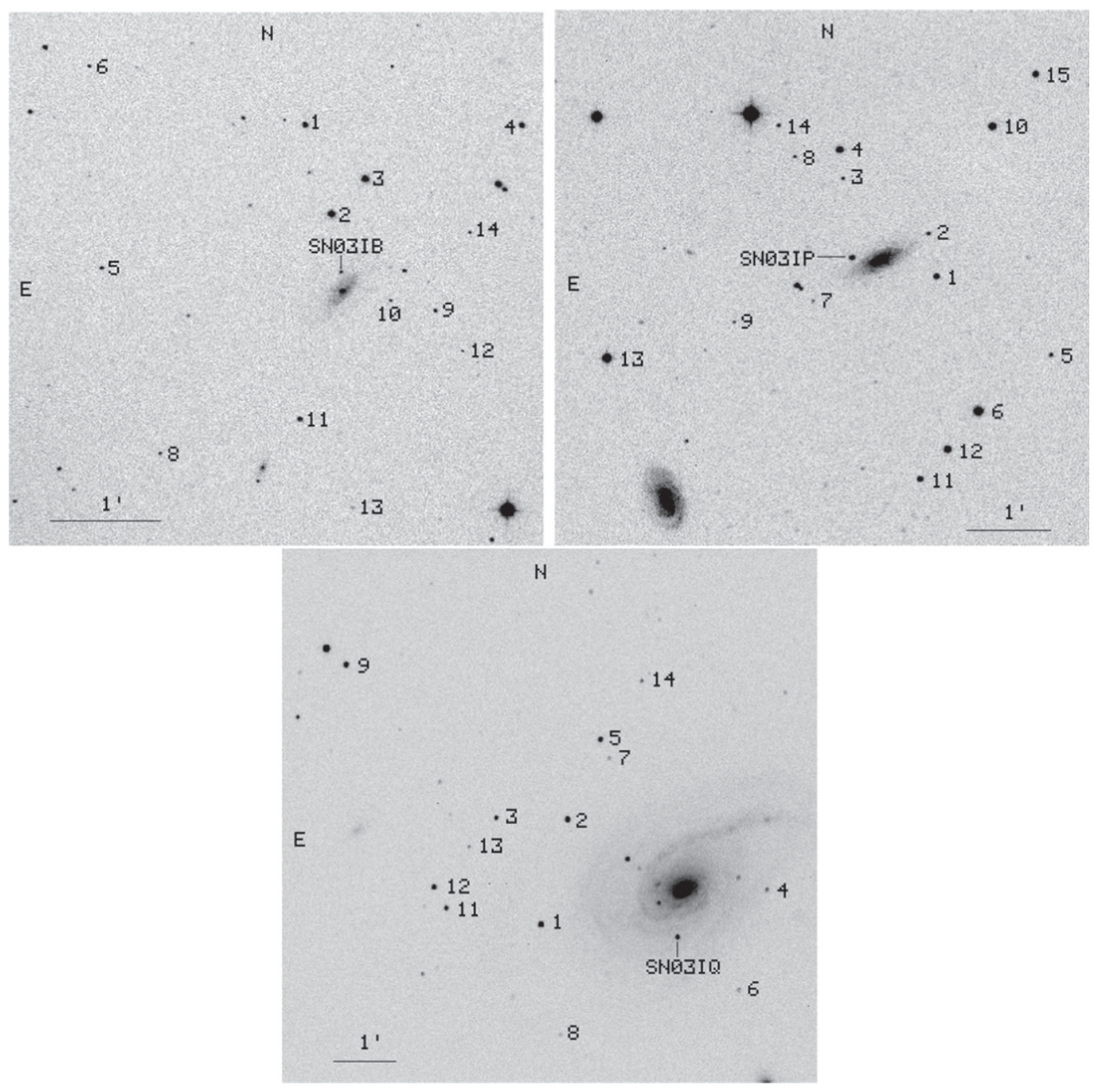

Figure 2. (Continued.)

Table 4

UBVRIZ Photometric Sequences

\begin{tabular}{|c|c|c|c|c|c|c|c|}
\hline Star & $U$ & $B$ & V & $R$ & $I$ & $z$ & $\bar{n}$ \\
\hline \multicolumn{8}{|c|}{ SN 1986L } \\
\hline 1 & $\ldots$ & $13.530(020)$ & $12.970(010)$ & $\ldots$ & $\ldots$ & $\ldots$ & 12 \\
\hline 2 & $\ldots$ & $15.710(050)$ & $14.770(020)$ & $\ldots$ & $\ldots$ & $\ldots$ & 12 \\
\hline 3 & $\ldots$ & $17.930(050)$ & $17.160(020)$ & $\ldots$ & $\ldots$ & $\ldots$ & 12 \\
\hline 4 & $\ldots$ & $16.360(050)$ & $15.500(020)$ & $\ldots$ & $\ldots$ & $\ldots$ & 12 \\
\hline 5 & $\ldots$ & $13.970(020)$ & $13.390(010)$ & $\ldots$ & $\ldots$ & $\ldots$ & 12 \\
\hline \multicolumn{8}{|c|}{ SN 1991al } \\
\hline 1 & $\ldots$ & $17.908(008)$ & $17.036(012)$ & $16.560(008)$ & $16.074(010)$ & $\ldots$ & 5 \\
\hline 2 & $\ldots$ & $17.633(010)$ & $16.770(006)$ & $16.273(010)$ & $15.808(008)$ & $\ldots$ & 5 \\
\hline 3 & $\ldots$ & $17.428(008)$ & $16.619(011)$ & $16.144(012)$ & $15.710(009)$ & $\ldots$ & 5 \\
\hline 4 & $\ldots$ & $15.390(009)$ & $14.427(010)$ & $13.889(015)$ & $13.391(009)$ & $\ldots$ & 5 \\
\hline 5 & $\ldots$ & $19.075(015)$ & $18.358(007)$ & $17.939(011)$ & $17.522(016)$ & $\ldots$ & 5 \\
\hline 6 & $\ldots$ & $18.846(012)$ & $18.365(006)$ & $18.039(007)$ & $17.676(012)$ & $\ldots$ & 5 \\
\hline 7 & $\ldots$ & $18.371(012)$ & $17.332(016)$ & $16.682(009)$ & $16.098(009)$ & $\ldots$ & 5 \\
\hline 8 & $\ldots$ & $16.436(010)$ & $15.558(010)$ & $15.058(012)$ & $14.572(013)$ & $\ldots$ & 5 \\
\hline 9 & $\ldots$ & $15.799(006)$ & $14.965(007)$ & $14.490(012)$ & $14.026(005)$ & $\ldots$ & 5 \\
\hline
\end{tabular}

Note. Uncertainties given in parenthesis in thousandths of a magnitude. $n$ is the number of nights on which each star was observed. (This table is available in its entirety in machine-readable form.) 
Table 5

UBVRIZ Photometry for 51 Type II Supernovae

\begin{tabular}{|c|c|c|c|c|c|c|c|}
\hline $\mathrm{JD}-2,400,000$ & $U$ & $B$ & $V$ & $R$ & $I$ & $z$ & Telescope \\
\hline \multicolumn{8}{|c|}{ SN 1986L } \\
\hline 46712.4 & $\ldots$ & $13.836(015)$ & $14.013(017)$ & $\ldots$ & $\ldots$ & $\ldots$ & CTIO-0.9 m \\
\hline 46712.4 & $\ldots$ & $13.815(015)$ & $13.940(017)$ & $\ldots$ & $\ldots$ & $\ldots$ & CTIO-4 m \\
\hline 46714.3 & $\ldots$ & $13.771(015)$ & $13.756(017)$ & $\ldots$ & $\ldots$ & $\ldots$ & СТIO-0.9 m \\
\hline 46715.3 & $\ldots$ & $13.712(015)$ & $13.711(017)$ & $\ldots$ & $\ldots$ & $\ldots$ & CTIO-0.9 m \\
\hline 46716.2 & $\ldots$ & $13.764(015)$ & $13.683(017)$ & $\cdots$ & $\cdots$ & $\ldots$ & CTIO- $0.9 \mathrm{~m}$ \\
\hline \multicolumn{8}{|c|}{ SN 1991al } \\
\hline 48455.67 & $\ldots$ & $16.608(018)$ & $\ldots$ & $16.230(015)$ & $\ldots$ & $\ldots$ & СТIO-0.9 m \\
\hline 48458.77 & $\ldots$ & $16.660(018)$ & $16.570(016)$ & $16.286(014)$ & $16.126(015)$ & $\ldots$ & CTIO-0.9 m \\
\hline 48459.60 & $\ldots$ & $16.664(018)$ & $16.604(016)$ & $16.300(014)$ & $16.138(015)$ & $\ldots$ & CTIO-0.9 m \\
\hline 48478.65 & $\ldots$ & $17.703(012)$ & $16.907(011)$ & $16.467(010)$ & $16.342(011)$ & $\ldots$ & СТIO-4 m \\
\hline 48490.70 & $\ldots$ & $\ldots$ & $17.067(014)$ & $\ldots$ & $16.380(015)$ & $\ldots$ & СТIO-0.9 m \\
\hline 48499.56 & $\ldots$ & $\ldots$ & $17.195(030)$ & $16.731(014)$ & $16.465(015)$ & $\ldots$ & CTIO-0.9 m \\
\hline 48508.63 & $\ldots$ & $18.702(018)$ & $17.378(016)$ & $16.848(014)$ & $16.568(015)$ & $\ldots$ & СТIO-0.9 m \\
\hline 48537.65 & $\ldots$ & $20.683(141)$ & $19.206(037)$ & $18.333(023)$ & $17.842(032)$ & $\ldots$ & CTIO-0.9 m \\
\hline 48545.53 & $\ldots$ & $20.864(349)$ & $19.237(065)$ & $\ldots$ & $\ldots$ & $\ldots$ & CTIO- $0.9 \mathrm{~m}$ \\
\hline 48546.52 & $\ldots$ & $20.984(200)$ & $19.309(041)$ & $\ldots$ & $\ldots$ & $\ldots$ & СТIO-0.9 m \\
\hline 48547.52 & $\ldots$ & $21.158(169)$ & $19.292(043)$ & $\ldots$ & $\ldots$ & $\ldots$ & CTIO-0.9 m \\
\hline
\end{tabular}

Note. Uncertainties given in parenthesis in thousandths of a magnitude.

(This table is available in its entirety in machine-readable form.)

average magnitudes are slightly lower $(\sim 0.7 \mathrm{mag})$ than those previously published, but still consistent within the uncertainties. While only a few objects peak at magnitudes brighter than the range covered by the $1 \sigma$ strip (including $\mathrm{SNe} 2003 \mathrm{bg}$ ), a higher number with low-luminosity appear below the strip. The range of magnitudes at peak span $\sim 4.5 \mathrm{mag}$ in all bands, from SN 1999br being the faintest and SN 2003eg the brightest. While the broad-line Type IIb SN 2003bg is one of the brightest objects in all bands, with data near maximum $(B, V, I)$, the other SNe IIn and 1987A-like objects have brightness around the average.

In Figure 7 we show the BVRI-band light curves referenced to the epoch of maximum brightness and normalized to peak magnitude. Once the differences in maximum brightness among SNe II are removed, these panels show how other light-curve parameters compare, such as the duration of the plateau, the post-maximum brightness decline, or the slope of the radioactive tail. As pointed out by Anderson et al. (2014), with the $V$-band light curves of the CSP sample, and the sample recently published by Sanders et al. (2015), we do not distinguish two separate groups among the diversity of light curves. On the other hand, our findings rather show a continuum in all the parameters listed above, and in all the optical bands presented here.

\subsection{Brightness Decline}

We measure the brightness decline parameter, $s$, defined as the decline rate in magnitudes per 100 days of the postmaximum light curve until the end of the plateau. This is similar to the $s 1$ and $s 2$ parameters defined by Anderson et al. (2014). For the reddest bands, the first phase of brightness decline after maximum right before entering into the recombination phase, $s 1$, is not clearly seen. Therefore, $s$ is adopted instead of the $s 2$ parameter in order to compare multiband observations in a systematic way. The exact details of this parameter and its behavior in different bands will be a matter of future work.

To measure $s$, it was first necessary to define the epoch at which the plateau ends for each band separately. Similar to Anderson et al. (2014), we set this as the epoch at the latest phases of the plateau at which the brightness deviates more than $0.1 \mathrm{mag}$ from the linear fits. We stress that (i) for a single $\mathrm{SN}$, this epoch is statistically the same in all bands, and (ii) their distribution is similar for all bands (although the average increases slightly for redder bands) and peaks at $77.5( \pm 26.3)$ days after the explosion epoch. Photometric decline rates are all measured by fitting a straight line to the defined phase, taking into account photometric errors. For those objects for which the end of the plateau in the $U$ band cannot be defined, we take that epoch from other bands.

The average values for the decline rates for all SNe II in our sample where the measurement can be confidently performed are: $\left\langle s_{U}\right\rangle=8.06 \mathrm{mag} 100 \mathrm{~d}^{-1}(\sigma=1.87,6 \mathrm{SNe}) ;\left\langle s_{B}\right\rangle=3.17 \mathrm{mag}$ $100 \mathrm{~d}^{-1}(\sigma=1.29, \quad 45 \quad \mathrm{SNe}) ; \quad\left\langle s_{V}\right\rangle=1.53 \mathrm{mag} \quad 100 \mathrm{~d}^{-1}$ $(\sigma=0.91,45 \mathrm{SNe}) ;\left\langle s_{R}\right\rangle=0.92 \mathrm{mag} 100 \mathrm{~d}^{-1}(\sigma=0.76,22$ $\mathrm{SNe}) ;\left\langle s_{I}\right\rangle=0.65 \mathrm{mag} 100 \mathrm{~d}^{-1}(\sigma=1.01,43 \mathrm{SNe})\left(\left\langle s_{z}\right\rangle\right.$ is not reported since there are not enough data to do statistics). Similar to the absolute magnitude distributions discussed above, SNe decline steeper in the bluer bands and the decline gets shallower in the redder bands.

Peculiar SNe, IIn and IIb SNe, have not been considered in the measurement of the average values since their light curves present different morphologies that are not described well by this characterization.

\subsection{Peak Magnitude and Brightness Decline Relation}

In this section we study the relation between the two parameters measured in the previous sections: the peak absolute magnitude and the brightness decline rate. This correlation holds promise for the standardization of the SN II 

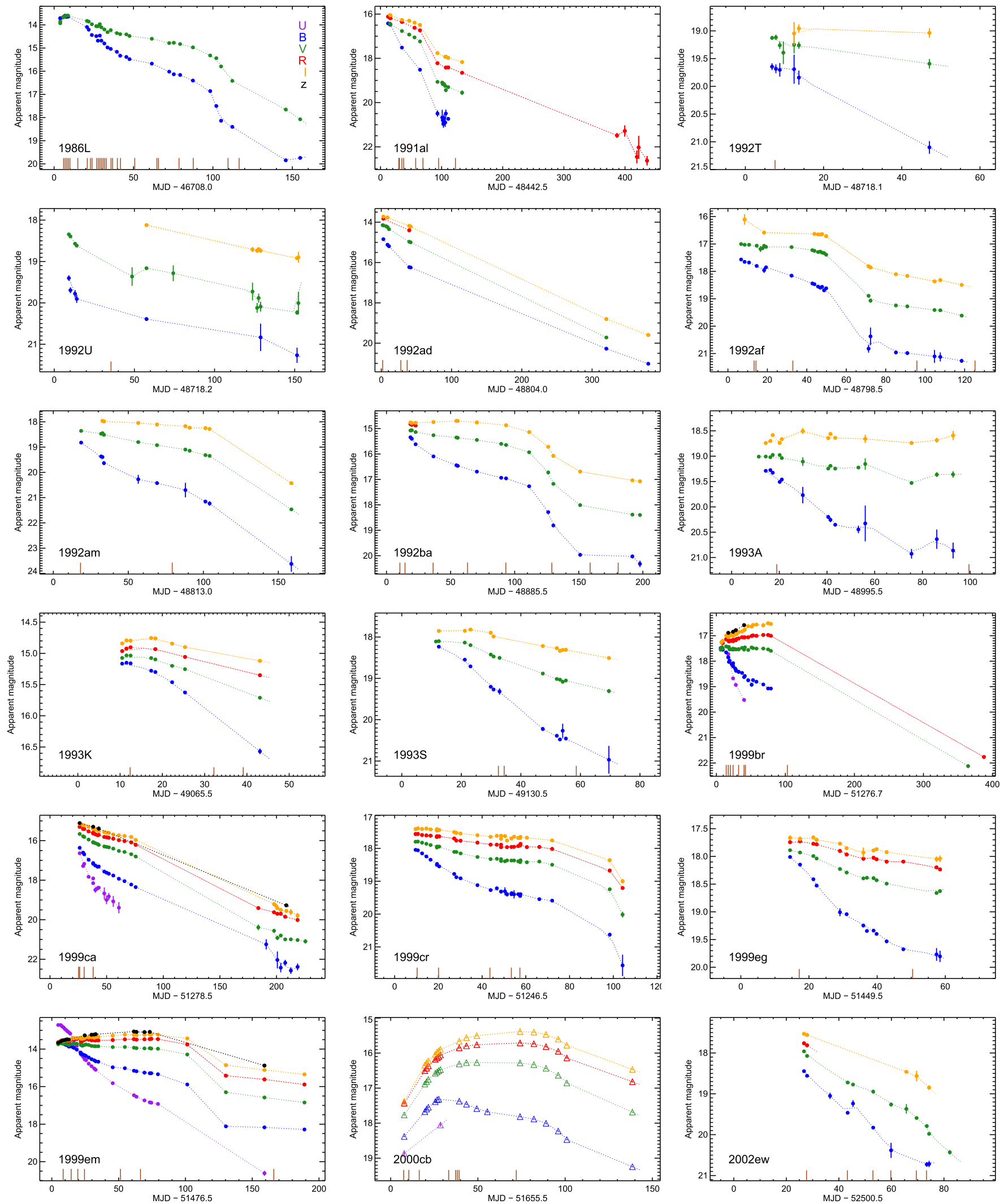

Figure 3. MW Extinction-corrected apparent UBVRIz light curves of the 51 SNee II. The explosion epoch in each panel is that estimated in C. Gutiérrez et al. (2016, in preparation). Photometric errors are plotted and are usually smaller than the symbol. Type IIb, IIn, and SN 1987A-like SNee II are plotted with unfilled triangles. Lines correspond to spline fits of the data. Vertical brown tick marks represent the epochs of the available spectra. 

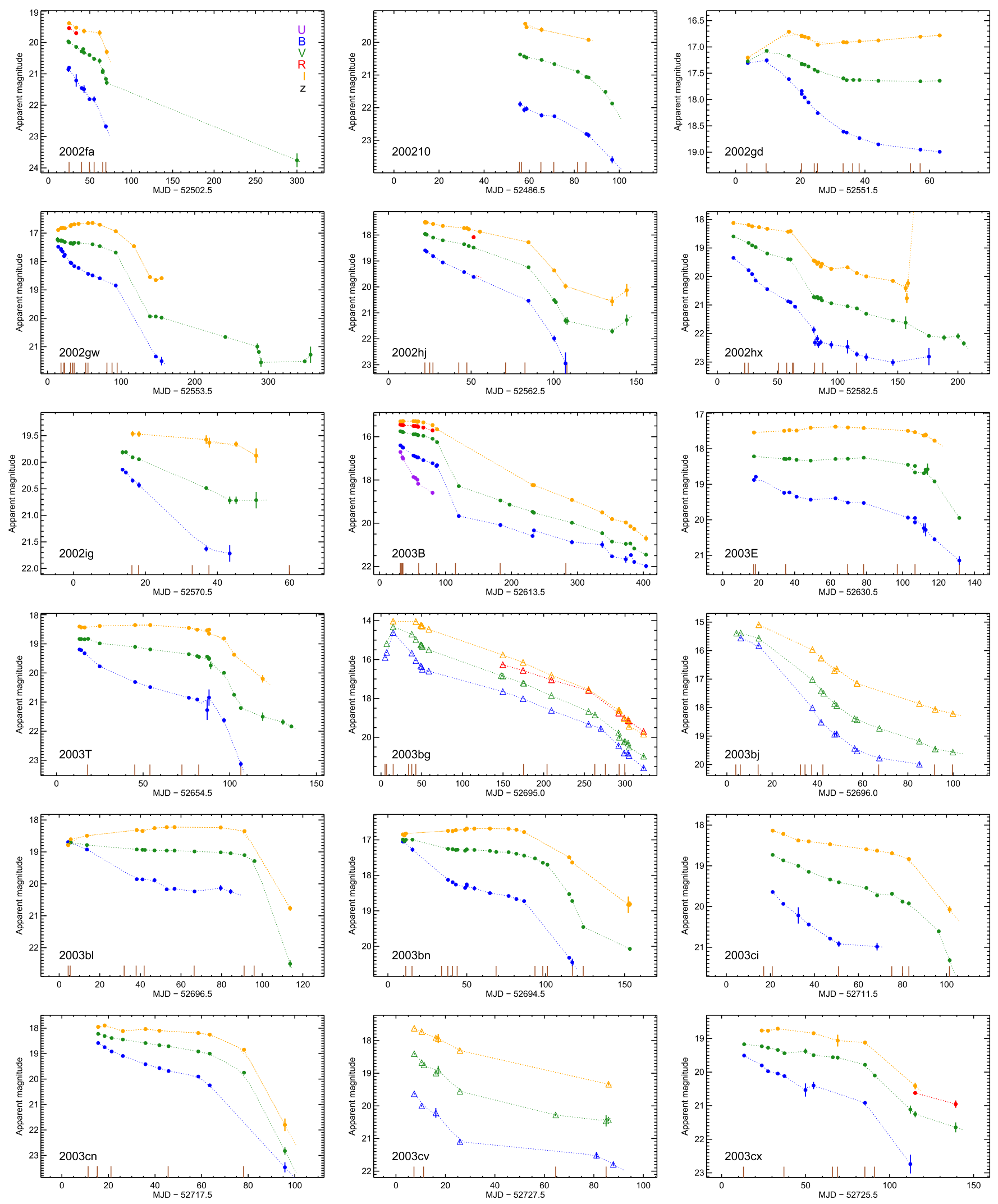

Figure 3. (Continued.) 

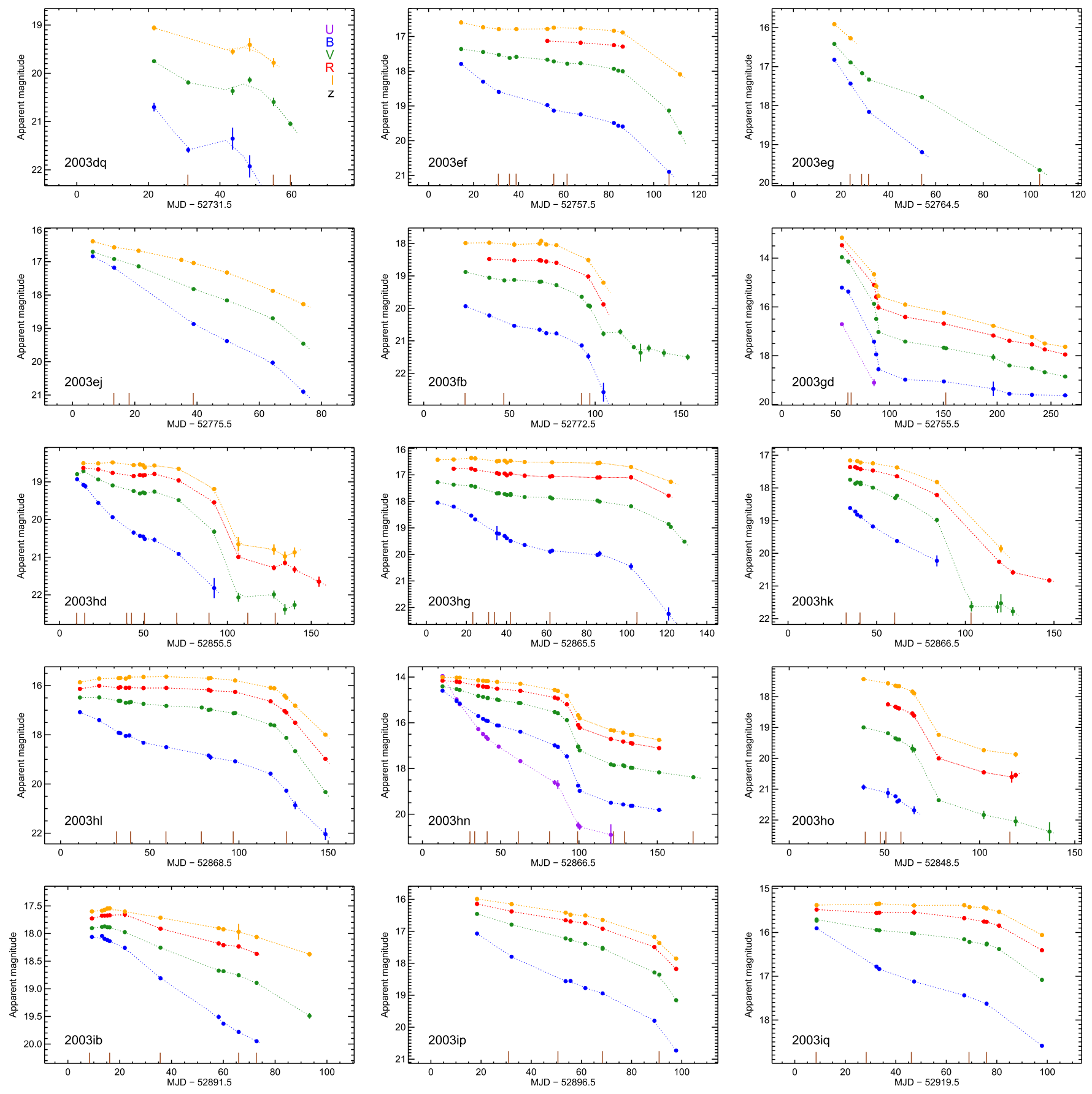

Figure 3. (Continued.)

absolute peak magnitudes and may enable their use as distance indicators for cosmology (Anderson et al. 2014), similar to the luminosity decline-rate relation used for $\mathrm{SNe}$ Ia (Phillips 1993b).

Here we exclude from our sample of $51 \mathrm{SNe}$ I the four IIb, IIn and SN 1987A-like events. To improve the statistics and the significance of the results presented below we expand our sample of $47 \mathrm{SNe}$ II to 114 by including $67 \mathrm{SNe}$ II for which photometry is available in the literature, as listed in Table 6.

Figure 8 presents histograms of the distributions of decline rates, $s$, in each band together with their median values for the complete sample. The same trends described in Section 3.4 are recovered: bluer bands show higher $s$ values, and the median values (represented in dashed vertical lines in the figure) are lower for redder bands.

In the left panel of Figure 9, $M_{\max }$ is plotted versus $s$. Filled circles are $\mathrm{SNe}$ presented in this paper and empty circles are objects from the literature. These parameters show a trend in the sense that lower luminosity SNe decline more slowly, while more luminous events declining more rapidly. This behavior was previously reported in the $V$-band (Anderson et al. 2014), and it is presented here for the first time for the UBVRI-bands 

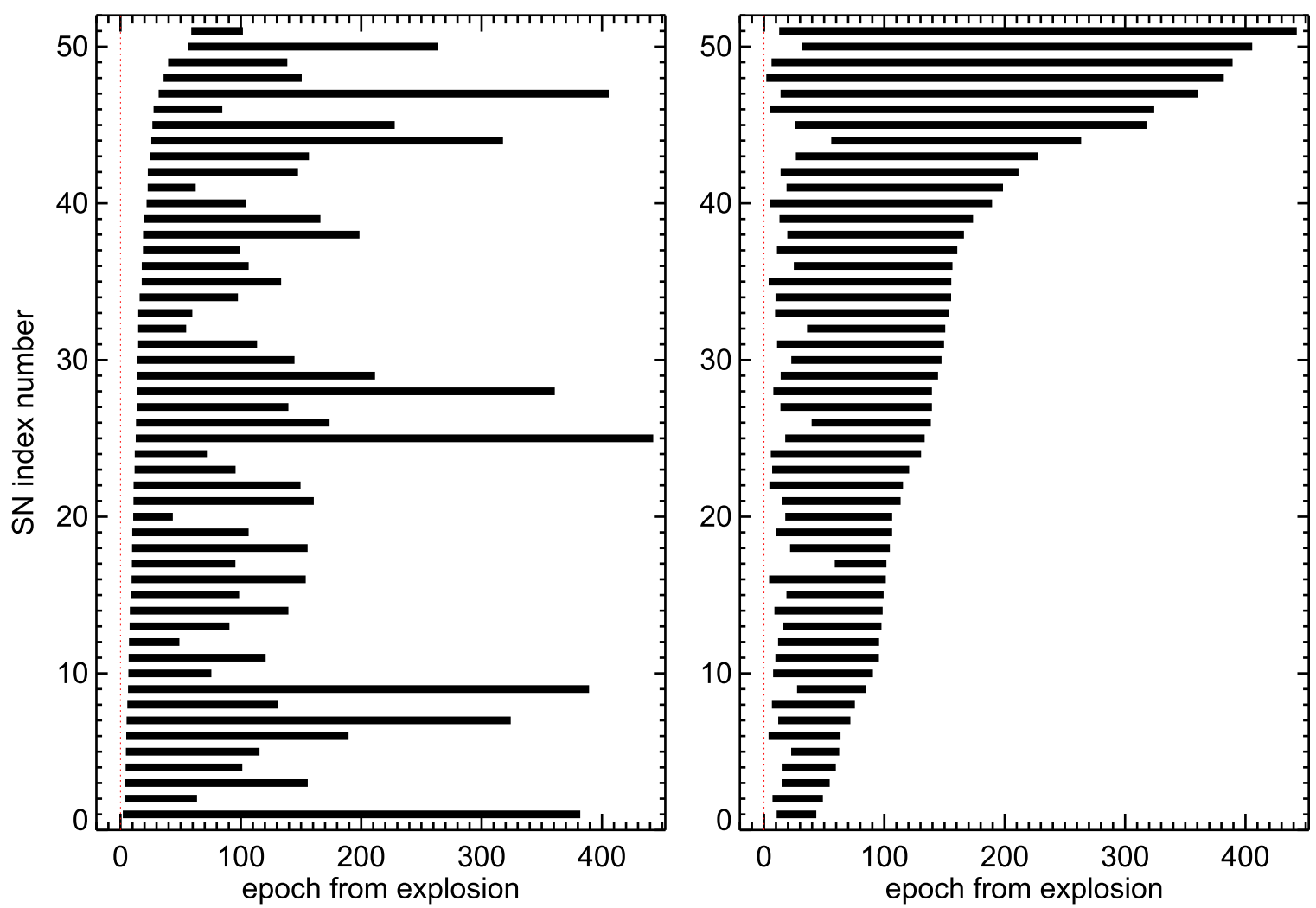

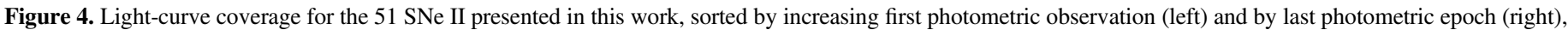

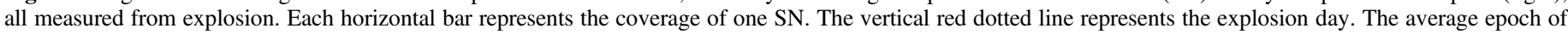
the first observation is $14.7 \pm 11.1$ days (median 11.7 days) and the last observation is $158.2 \pm 98.7$ days (median 139.5 days).

light curves. We performed linear fits to the data in each band and we found that the one showing the best correlation is the $B$ band $(r=-0.59, N=97)$. This is contradictory to the insignificant correlation found by Pejcha \& Prieto (2015a), but their result can be due to the small size of their sample.

For those objects for which the measurement of the plateau duration is possible, we show in the right panel of Figure 9 its relation with the $s$ parameter. Plateau durations cover a range from $\sim 20$ to $\sim 80$ days in all bands, with some SNe having shorter plateaus in the bluer bands, and some with higher values for redder bands. SNe with deeper declines (higher $s$ ) are found also to have shorter plateaus, and SNe whom decline in brightness more slowly (lower $s$ ) have longer plateaus. Linear fits to the BVRI data separately give similar slopes of -0.03 (mag $100 \mathrm{~d}^{-1}$ per day), with increasing correlation factors from -0.6 to -0.8 for bluer to redder bands.

Both the luminosity and the plateau duration are related to $s$, in a way that $\mathrm{SNe}$ declining faster have shorter plateaus and brighter magnitudes (see Figure 9). According to Kasen \& Woosley (2009; see also Sukhbold et al. 2015) models, these two parameters basically depend on the kinetic energy of the explosion and the mass of the ejecta (see also Popov 1993). ${ }^{29}$ For larger and/or denser $\mathrm{H}$ layers, a higher fraction of energy is lost in the diffusion of the radiation through the envelope, the radiation is trapped for a longer time (thus longer duration plateau phases), and less energy/radiation escapes and

\footnotetext{
${ }^{29}$ Note that the mass of radioactive ${ }^{56} \mathrm{Ni}$ synthesized in the explosion extends the plateau in time by a few percent (Kasen \& Woosley 2009). It also powers the luminosity after the recombinations phase, and its total mass has been shown to correlate well also with the plateau luminosity (Hamuy 2003a; Pejcha \& Prieto 2015a).
}

contributes to the luminosity (Blinnikov \& Bartunov 1993). The observed relations between luminosity, plateau duration, and decline rate (s) indicate that SNee exploding with higher kinetic energies are those resulting from progenitors with smaller and/or less denser $\mathrm{H}$ envelope masses at the explosion. This is in agreement with the current view of massive stars evolution, where the progenitors of core collapse $\mathrm{SNe}$ with reduced $\mathrm{H}$ envelopes are stars with larger zero-age main sequence masses that have experienced a higher degree of mass-loss prior to explosion (Heger et al. 2003).

Finally, the wide range of plateau durations, decline rates, and peak luminosities can also be interpreted as a clear indication of a continuity in the $\mathrm{SNe}$ II class.

\section{SUMMARY AND CONCLUSIONS}

This paper presents a sample of multi-band, visualwavelength light curves of 51 SNe II observed from 1986 to 2003 in the course of four different surveys: the Cerro Tololo Supernova Survey, the C\&T, the SOIRS, and the CATS. Nearinfrared photometry and optical spectroscopy of this set of SNe II will be published in two companion papers.

After determining their explosion dates and correcting all photometry for Galactic extinction and time dilation, we investigated their color behavior in different bands, and measured their peak absolute magnitudes and the brightness decline in the recombination phase in all bands. No evidence of two separate families (SNe IIP and SNe IIL) can be seen in our results, confirming previous reports that there is a continuity in SNe II characteristics. 

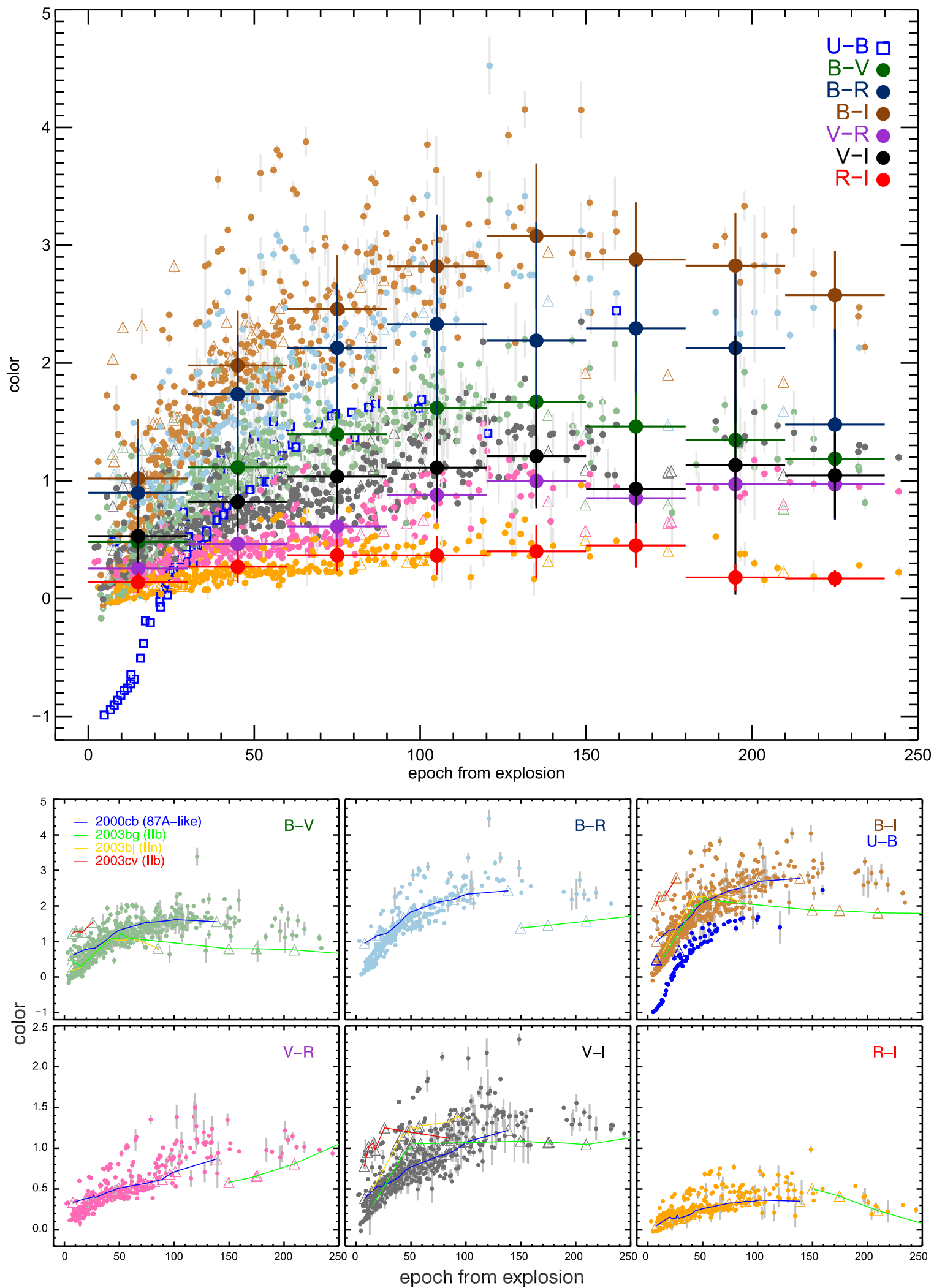

Figure 5. Color evolution diagrams. Top: small dots represent individual measurements and big dots indicate average colors in bins of 30 days. All of them increase until reaching a maximum around 100-150 days from explosion, and then saturate or start decreasing. Blue empty squares are the $(U-B)$ color data. Bottom: individual color panels. Color measurements of the SN 1987A-like, IIb, and IIn are plotted with empty triangles, and in $(B-I)$ they are shown in solid lines.

All color curves grow steadily redder during the first few weeks due to a decrease in surface temperature, and reach a maximum around $\sim 100-150$ days, followed by a shallow color decrease. $(U-B)$ colors are found to begin with negative values around maximum, followed by a rapid increase to redder colors owing to the temperature decline (cooling) and the increasingly higher line blanketing toward shorter wavelengths. 

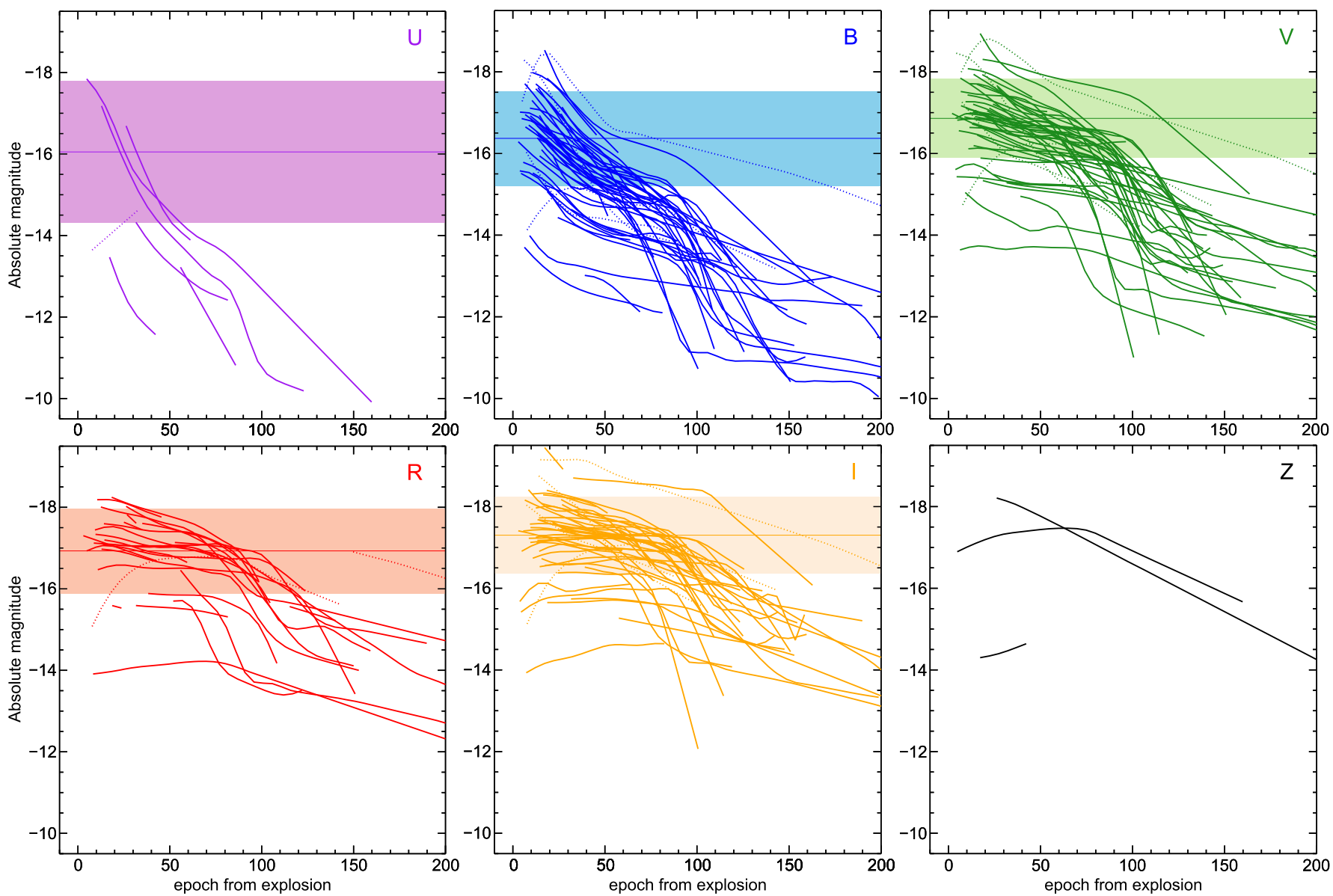

Figure 6. Absolute magnitude light curves for all SNe presented here overplotted in separate panels by different bands. Curves have been smoothed using a third order spline polynomials. Type IIb, IIn, and peculiar SNe II are shown with dotted lines. The horizontal lines and the colored stripes correspond to the peak average absolute magnitudes and their $1 \sigma$ deviation.
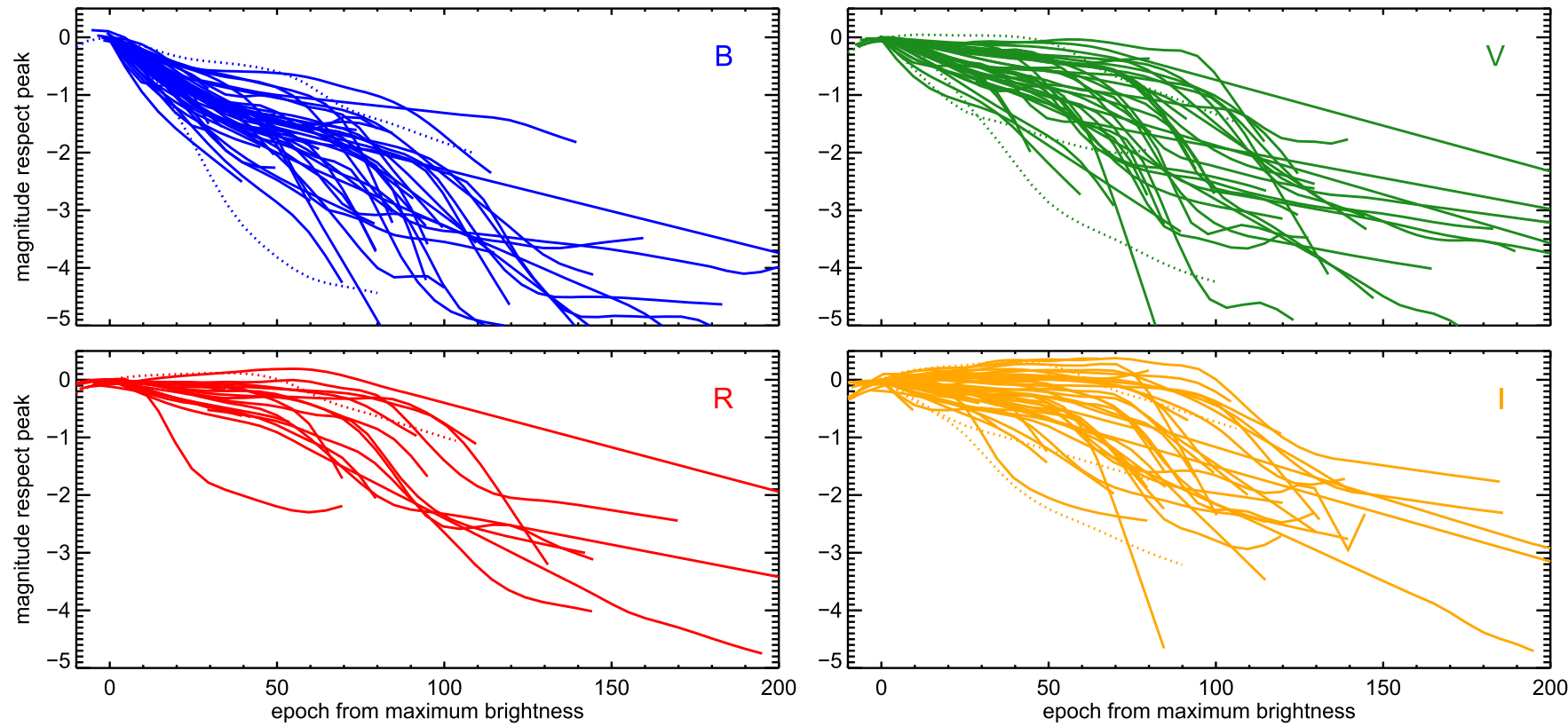

Figure 7. Magnitude light curves referenced to the epoch of the maximum brightness and normalized to the peak. Type IIb, IIn, and peculiar SNe II are plotted with dotted lines. All panels show a continuous distribution between fast and slow decliners in all bands. 
Table 6

Type II Supernovae Used in Figure 8

\begin{tabular}{|c|c|c|}
\hline SN Name & Bands & Photometry Reference \\
\hline $1968 \mathrm{~L}$ & $U B V$ & Wood \& Andrews (1974) \\
\hline 1970G & $U B V$ & Barbon et al. (1973), Winzer (1974) \\
\hline 1973R & $B V$ & Ciatti \& Rosino (1977) \\
\hline 1986I & $V R I$ & Pennypacker et al. (1989) \\
\hline 1988A & $B V R$ & Benetti et al. (1991), Turatto et al. (1993) \\
\hline $1988 \mathrm{H}$ & $B V R$ & Turatto et al. (1993) \\
\hline 1989C & $B V R$ & Turatto et al. (1993) \\
\hline 1990E & $B V R I$ & Schmidt et al. (1993), Benetti et al. (1994) \\
\hline $1990 \mathrm{~K}$ & $B V R I$ & Cappellaro et al. (1995) \\
\hline $1991 \mathrm{G}$ & $V R I$ & Blanton et al. (1995) \\
\hline $1992 \mathrm{H}$ & $B V R$ & Clocchiatti et al. (1996) \\
\hline $1993 \mathrm{G}$ & $B V$ & Tsvetkov (1994) \\
\hline $1994 N$ & $B V R$ & Pastorello et al. (2004) \\
\hline 1995ad & $B V R I$ & Inserra et al. (2013) \\
\hline 1996W & UBVRI & Inserra et al. (2013) \\
\hline 1998S & $B V R$ & Liu et al. (2000) \\
\hline 1999bg & $B V I$ & Faran et al. (2014b) \\
\hline 1999co & $B V I$ & Faran et al. (2014a) \\
\hline 1999eu & UBVRI & Pastorello et al. (2004) \\
\hline 1999gi & $B V R I$ & Leonard et al. (2002) \\
\hline $2000 \mathrm{dc}$ & $B V R I$ & Faran et al. (2014a) \\
\hline 2000dj & $B V I$ & Faran et al. (2014b) \\
\hline $2001 X$ & $B V R I$ & Tsvetkov (2006), Faran et al. (2014b) \\
\hline $2001 \mathrm{bq}$ & $B V I$ & Faran et al. (2014b) \\
\hline $2001 \mathrm{~cm}$ & $B V I$ & Faran et al. (2014b) \\
\hline 2001cy & $B V R I$ & Faran et al. (2014a) \\
\hline $2001 \mathrm{dc}$ & $B V R I$ & Pastorello et al. (2004) \\
\hline 2001do & $B V R I$ & Faran et al. (2014a) \\
\hline $2001 \mathrm{fa}$ & $B V R I$ & Faran et al. (2014a) \\
\hline $2002 \mathrm{hh}$ & $V R I$ & $\begin{array}{l}\text { Pozzo et al. (2006), Tsvetkov et al. (2007), Faran } \\
\text { et al. (2014b) }\end{array}$ \\
\hline $2003 Z$ & $B V R I$ & Spiro et al. (2014), Faran et al. (2014b) \\
\hline $2003 \mathrm{hf}$ & $B V R I$ & Faran et al. (2014a) \\
\hline $2004 \mathrm{~A}$ & $B V R I$ & Hendry et al. (2006), Tsvetkov (2008) \\
\hline 2004dj & $U B V R I$ & $\begin{array}{l}\text { Chugai et al. (2005), Vinkó et al. (2006) } \\
\text { Zhang et al. (2006); Tsvetkov et al. (2008) }\end{array}$ \\
\hline 2004eg & $B V R I$ & Spiro et al. (2014) \\
\hline 2004et & $U B V R I$ & Misra et al. (2007), Faran et al. (2014b) \\
\hline 2005ay & $U B V R I$ & Tsvetkov (2006), Faran et al. (2014b) \\
\hline $2005 \mathrm{cs}$ & $U B V R I$ & $\begin{array}{l}\text { Tsvetkov et al. (2006), Dessart et al. (2008), } \\
\text { Pastorello et al. (2009) }\end{array}$ \\
\hline $2005 \mathrm{dq}$ & $B V R I$ & $\begin{array}{l}\text { Pritchard et al. (2014), Faran et al. (2014b) } \\
\text { Faran et al. (2014a) }\end{array}$ \\
\hline $2006 \mathrm{bp}$ & $U B V$ & Dessart et al. (2008), Pritchard et al. (2014) \\
\hline 2006ov & $B V R I$ & Spiro et al. (2014) \\
\hline $2007 \mathrm{ck}$ & $U B V$ & Pritchard et al. (2014) \\
\hline $2007 \mathrm{fz}$ & $B V R I$ & Faran et al. (2014a) \\
\hline $2008 \mathrm{fq}$ & $B V R I$ & Faran et al. (2014a) \\
\hline $2008 \mathrm{ij}$ & $U B V$ & Pritchard et al. (2014) \\
\hline $2008 \mathrm{jb}$ & $V I$ & Prieto et al. (2012) \\
\hline 2009at & $U B V$ & Prieto et al. (2012) \\
\hline 2009bw & $U B V R I$ & Inserra et al. (2012) \\
\hline 2009dd & UBVRI & Prieto et al. (2012), Inserra et al. (2013) \\
\hline 2009js & $B V R I$ & Gandhi et al. (2013) \\
\hline $2009 \mathrm{kr}$ & $U B V$ & Prieto et al. (2012) \\
\hline 2009md & UBVRI & Fraser et al. (2011) \\
\hline $2010 \mathrm{~F}$ & $U B V$ & Prieto et al. (2012) \\
\hline 2010aj & $U B V R I$ & Inserra et al. (2013) \\
\hline $2010 \mathrm{gs}$ & $U B V$ & Prieto et al. (2012) \\
\hline 2010id & UBVRI & Gal-Yam et al. (2011) \\
\hline 2011cj & $U B V$ & Prieto et al. (2012) \\
\hline $2012 \mathrm{~A}$ & $U B V R I$ & Prieto et al. (2012), Tomasella et al. (2013) \\
\hline 2012ak & $U B V$ & Prieto et al. (2012) \\
\hline
\end{tabular}

Table 6

(Continued)

\begin{tabular}{lcc}
\hline \hline SN Name & Bands & Photometry Reference \\
\hline 2012aw & UBVRI & Prieto et al. (2012), Bose et al. (2013), Dall'Ora \\
& & et al. (2014) \\
$2012 \mathrm{ec}$ & $B V R I$ & Barbarino et al. (2015), Jerkstrand et al. (2015) \\
$2013 \mathrm{ab}$ & $U B V R I$ & Barbarino et al. (2015) \\
$2013 \mathrm{am}$ & $U B V R I$ & Zhang et al. (2014) \\
$2013 \mathrm{by}$ & $U B V$ & Valenti et al. (2014) \\
2013ej & $U B V R I$ & Valenti et al. (2014), Richmond (2014) \\
ASASSNe- & $U B V$ & Holoien et al. (2014) \\
13co & & Gall et al. (2015) \\
LSQ13cuw & $V$ & \\
\hline
\end{tabular}

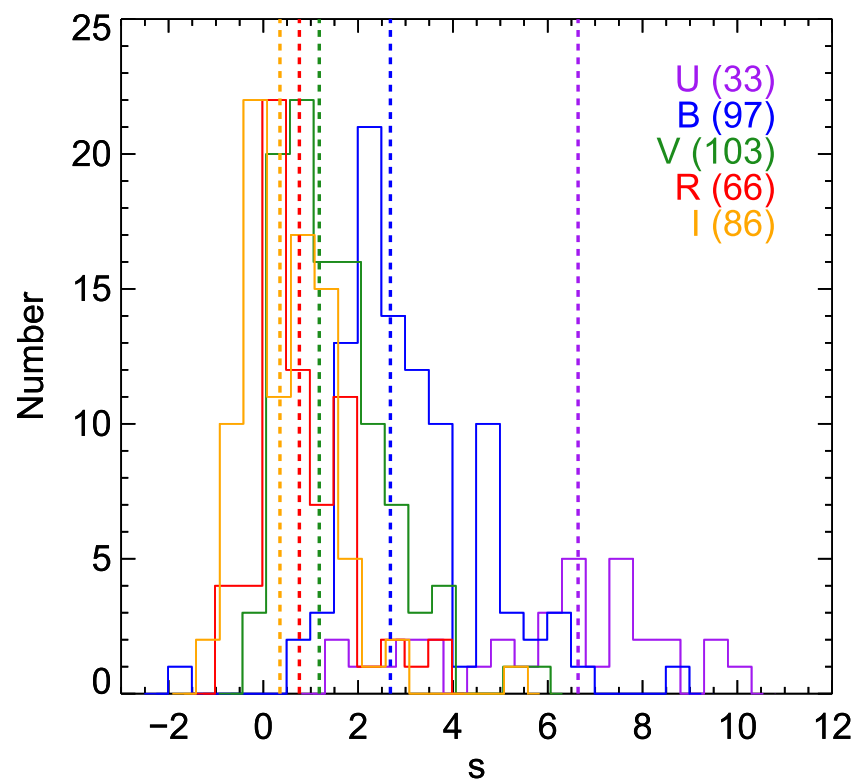

Figure 8. Distribution of the slopes of the plateau in each filter for the expanded sample of $114 \mathrm{SNe}$ II. The median of the distribution decreases with redder filters.

For a given color index, the scatter among different $\mathrm{SNe}$ increases for color indices involving bluer bands, supporting the idea that the color diversity could be caused by host-galaxy dust extinction. Going a step further and assuming that all $\mathrm{SNe}$ have similar intrinsic color curves, we note that $\mathrm{SNe}$ with higher excess in one color index also have higher excess in other color indices, whereas the bluest $\mathrm{SNe}$ appear blue in all color indices, lending support to the idea that the color excess is an indication of host-galaxy dust extinction. However, it is possible that part of the color diversity could be due to intrinsic effects. The low luminosity SNe $1999 \mathrm{br}$ is a clear example of an intrinsically red SNe. In a future paper (de Jaeger et al.) we will address this issue.

With all the available MW extinction-corrected absolute magnitude light curves we find a wide range of magnitudes and light curve morphologies in all UBVRIz bands. We measured absolute peak magnitudes, finding the following mean values: $\left\langle U_{\max }\right\rangle=-16.06 \pm 1.74 ;\left\langle B_{\max }\right\rangle=-16.43 \pm 1.19 ;\left\langle V_{\max }\right\rangle=-$ $16.89 \pm 0.98 ;\left\langle R_{\max }\right\rangle=-16.96 \pm 1.03 ;\left\langle I_{\max }\right\rangle=-17.38 \pm 0.95$. Only a few outliers with peak magnitudes brighter and fainter than $1 \sigma$ of the distribution are found.

We defined the $s$ parameter, which measures the brightness decline rate from maximum light through the end of the 

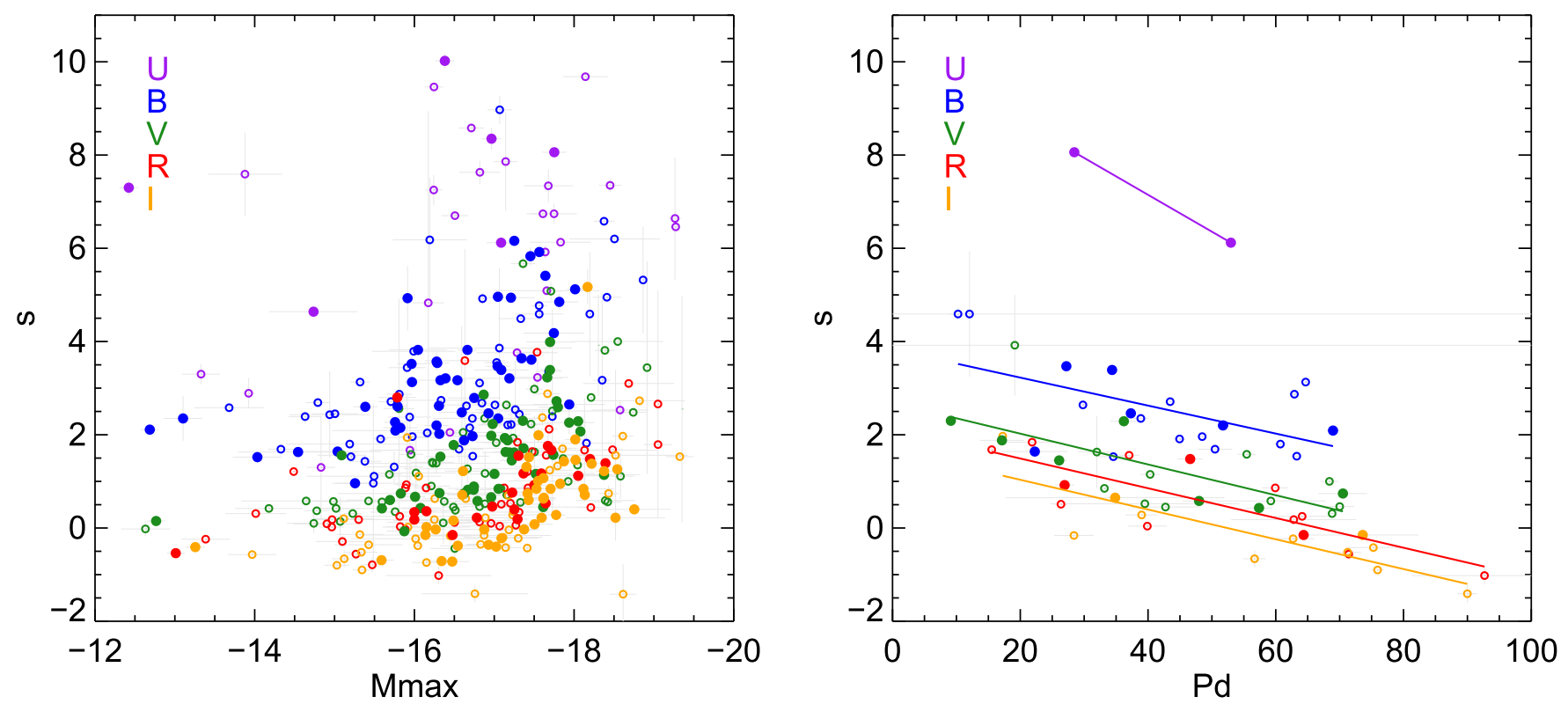

Figure 9. Left: slope of the plateau vs. peak absolute magnitude for all UBVRI bands. Filled circles correspond to the SNe II presented in this work, while empty circles are other objects from the literature. Peculiar objects such as SNe IIb, IIn, and 1987A-like SNe are excluded from this analysis. Right: relation between the plateau duration and the post-maximum brightness decline. A trend can be seen in all bands indicating shorter plateaus for faster declining SNe. Solid lines indicate linear fits to the points.

recombination phase, and found that this decline parameter is steeper in the blue bands than redder bands.

We added a set of 67 low- $z$ SNe II with publicly available photometry to study the absolute magnitude versus brightness decline parameter $s$ relation. From a total sample of 114 SNe II, we found a clear correlation in all bands, with the following characteristics: (1) more luminous $\mathrm{SNe}$ have steeper light curves; (2) the slope of the correlation decreases with increasing wavelength; and (3) the correlation is higher in the $B$ band. Finally, we also found a correlation between the $s$ parameter and the plateau duration, the latter being shorter (longer) for larger (smaller) $s$ values.

The complete set of photometry is available electronically ${ }^{30}$ or can be requested from the authors. Each SN folder in the tarball includes an info file containing its name, subtype, redshift, coordinates, host galaxy name, morphology, and MW extinction from Schlafly \& Finkbeiner (2011).

We acknowledge the contribution to the observations of Elisa Abedrapo, Maria Teresa Acevedo, Sandra dos Anjos, Roberto Avilés A., L. Felipe Barrientos, Timothy Ellsworth Bowers, Stephane Brillant, Pablo Candia, Sergio Castellón, Carlos Contreras, Arjun Dey, Vannessa Doublier, Jo Ann Eder, Jonathan Elias, Erica Ellingson, Wendy L. Freedman, Catharine Garmany, Ximena Gómez, Paul J. Green, Olivier R. Hainaut, Leonor Huerta, Daniel Kelson, Rebecca A. Koopmann, Arlo U. Landolt, Andrew Layden, Paul Martini, Philip Massey, Mario Mateo, Mauricio Navarrete, Edward W. Olszewski, Fernando Peralta, Joaquín Perez, Eric Persson, Tim Pickering, Miguel Roth, Eric P. Rubenstein, Maria Teresa Ruiz, Paul C. Schmidtke, Juan C. Seguel, Patrick Seitzer, Robert C. Smith, Ronaldo E. de Souza, Joao E. Steiner, Neil de Grasse Tyson, Stephanie Wachter, Ken-ichi Wakamatsu, Alistair Walker, Doug Welch, and Howard K.C. Yee. Support for L.G., M.H., T.M., S.G.G., and C.P.G. is provided by

\footnotetext{
${ }^{30}$ https://github.com/lgalbany/51_SNeII_LC
}

the Ministry of Economy, Development, and Tourism's Millennium Science Initiative through grant IC120009, awarded to The Millennium Institute of Astrophysics, MAS. L.G. and S.G.G. acknowledge support by CONICYT through FONDECYT grants 3140566 and 3130680, respectively. M.H. acknowledges support provided by Fondecyt grants 1920312 and 1060808, the Millennium Center for Supernova Science through grant P06-045-F funded by "Programa Bicentenario de Ciencia y Tecnología de CONICYT" and "Programa Iniciativa Científica Milenio del Ministerio de Economía," the Carnegie Postdoctoral Fellowship, and NASA through Hubble Fellowship grant HST-HF-01139.01-A, awarded by the Space Telescope Science Institute, which is operated by the Association of Universities for Research in Astronomy, Inc., for NASA, under contract NAS 5-26555. N.B.S. is thankful for support from the George P. and Cynthia Woods Mitchell Institute for Fundamental Physics and Astronomy. M.S. acknowledges the generous support provided by the Danish Agency for Science and Technology and Innovation, realized through a Sapere Aude Level 2 grant. A.L. was funded by grant No. CW-0004-85 from the Space Telescope Science Institute (STScI) at the time of observations. This work is based in part on observations at Cerro Tololo Inter-American Observatory, National Optical Astronomy Observatory, which is operated by the Association of Universities for Research in Astronomy (AURA) under a cooperative agreement with the National Science Foundation. This paper includes data gathered with the $6.5 \mathrm{~m}$ Magellan Telescopes located at Las Campanas Observatory, Chile. This work is based in part on observations made with the ESO Telescopes at the La Silla and Paranal Observatories under programmes 163.H-0285 and 164.H0376. We also acknowledge time allocations at the Steward Observatory of the University of Arizona. This research has made use of the NASA/IPAC Extragalactic Database (NED), which is operated by the Jet Propulsion Laboratory, California Institute of Technology, under contract with the National Aeronautics and Space Administration. 


\section{REFERENCES}

Anderson, J. P., González-Gaitán, S., Hamuy, M., et al. 2014, ApJ, 786, 67 Anderson, J. P., Habergham, S. M., James, P. A., \& Hamuy, M. 2012, MNRAS, 424, 1372

Barbarino, C., Dall'Ora, M., Botticella, M. T., et al. 2015, MNRAS, 448, 2312

Barbon, R., Ciatti, F., \& Rosino, L. 1973, A\&A, 29, 57

Benetti, S., Cappellaro, E., \& Turatto, M. 1991, A\&A, 247, 410

Benetti, S., Cappellaro, E., Turatto, M., et al. 1994, A\&A, 285, 147

Bersten, M. C., Benvenuto, O., \& Hamuy, M. 2011, ApJ, 729, 61

Bersten, M. C., \& Hamuy, M. 2009, ApJ, 701, 200

Blanton, E. L., Schmidt, B. P., Kirshner, R. P., et al. 1995, AJ, 110, 2868

Blinnikov, S. I., \& Bartunov, O. S. 1993, A\&A, 273, 106

Blondin, S., \& Tonry, J. L. 2007, ApJ, 666, 1024

Boles, T., \& Li, W. 2003, CBET, 41, 1

Bose, S., Kumar, B., Sutaria, F., et al. 2013, MNRAS, 433, 1871

Bouchet, P., della Valle, M., \& Melnick, J. 1991, IAUC, 5312, 2

Burrows, A. 2000, Natur, 403, 727

Cappellaro, E., Danziger, I. J., della Valle, M., Gouiffes, C., \& Turatto, M. 1995, A\&A, 293, 723

Cardelli, J. A., Clayton, G. C., \& Mathis, J. S. 1989, ApJ, 345, 245

Chassagne, R. 2003, IAUC, 8085, 1

Chieffi, A., Domínguez, I., Höflich, P., Limongi, M., \& Straniero, O. 2003, MNRAS, 345, 111

Chornock, R., Jha, S., Filippenko, A. V., \& Barris, B. 2002, IAUC, 8008, 2

Chugai, N. N., Fabrika, S. N., Sholukhova, O. N., et al. 2005, AstL, 31, 792

Ciatti, F., \& Rosino, L. 1977, A\&A, 56, 59

Clocchiatti, A., Benetti, S., Wheeler, J. C., et al. 1996, AJ, 111, 1286

Dall'Ora, M., Botticella, M. T., Pumo, M. L., et al. 2014, ApJ, 787, 139

della Valle, M., \& Bianchini, A. 1992, IAUC, 5558, 3

Dessart, L., Blondin, S., Brown, P. J., et al. 2008, ApJ, 675, 644

Dessart, L., Hillier, D. J., Waldman, R., \& Livne, E. 2013, MNRAS, 433, 1745

Elias-Rosa, N., Benetti, S., Marmo, C., et al. 2003, IAUC, 8187, 2

Evans, R., Bock, G., Krisciunas, K., \& Espinoza, J. 2003, IAUC, 8186, 1

Evans, R., McNaught, R., Cragg, T., \& Thompson, G. 1986, IAUC, 4260, 1

Evans, R., \& McNaught, R. H. 2003, IAUC, 8150, 2

Evans, R., \& Phillips, M. M. 1992, IAUC, 5625, 2

Evans, R., \& Quirk, S. 2003, IAUC, 8042, 1

Faran, T., Poznanski, D., Filippenko, A. V., et al. 2014a, MNRAS, 445,554

Faran, T., Poznanski, D., Filippenko, A. V., et al. 2014b, MNRAS, 442, 844 Filippenko, A. V. 1997, ARA\&A, 35, 309

Filippenko, A. V., \& Chornock, R. 2002, IAUC, 7988, 3

Filippenko, A. V., \& Foley, R. J. 2003, IAUC, 8214, 2

Filippenko, A. V., Foley, R. J., \& Serduke, F. J. D. 2003, IAUC, 8189, 2

Foley, R. J., Graham, J., Ganeshalingam, M., \& Filippenko, A. V. 2003, IAUC, 8060, 3

Fraser, M., Ergon, M., Eldridge, J. J., et al. 2011, MNRAS, 417, 1417

Frey, L. H., Fryer, C. L., \& Young, P. A. 2013, ApJL, 773, L7

Galbany, L., Stanishev, V., Mourão, A. M., et al. 2014, A\&A, 572, A38

Gall, E. E. E., Polshaw, J., Kotak, R., et al. 2015, A\&A, 582, A3

Gal-Yam, A., Kasliwal, M. M., Arcavi, I., et al. 2011, ApJ, 736, 159

Gandhi, P., Yamanaka, M., Tanaka, M., et al. 2013, ApJ, 767, 166

Ganeshalingam, M., Graham, J., Pugh, H., \& Li, W. 2003, IAUC, 8134, 1

Ganeshalingam, M., \& Li, W. 2003a, CBET, 15, 1

Ganeshalingam, M., \& Li, W. 2003b, IAUC, 8179, 2

Garnavich, P., \& Bass, E. 2003, IAUC, 8150, 3

Garnavich, P., Jha, S., Challis, P., et al. 1999, IAUC, 7143, 1

Graham, J., \& Li, W. 2002, IAUC, 8015, 1

Gutiérrez, C. P., Anderson, J. P., Hamuy, M., et al. 2014, ApJL, 786, L15

Hamuy, M. 1993a, IAUC, 5771, 1

Hamuy, M. 1993b, IAUC, 5823, 1

Hamuy, M. 2002a, IAUC, 7987, 2

Hamuy, M. 2002b, IAUC, 7968, 1

Hamuy, M. 2003a, ApJ, 582, 905

Hamuy, M. 2003b, IAUC, 8102, 4

Hamuy, M. 2003c, IAUC, 8117, 2

Hamuy, M. 2003d, IAUC, 8045, 3

Hamuy, M. 2004, in Measuring and Modeling the Universe, ed.

W. L. Freedman (Cambridge: Cambridge Univ. Press), 2

Hamuy, M., Deng, J., Mazzali, P. A., et al. 2009, ApJ, 703, 1612

Hamuy, M., Folatelli, G., Morrell, N. I., et al. 2006, PASP, 118, 2

Hamuy, M., Maza, J., \& Huerta, L. 2003a, IAUC, 8109, 1

Hamuy, M., Maza, J., Phillips, M. M., et al. 1993, AJ, 106, 2392

Hamuy, M., Morrell, N., \& Thomas-Osip, J. 2003b, IAUC, 8183, 2

Hamuy, M., Phillips, M., \& Thomas-Osip, J. 2003c, IAUC, 8088, 3
Hamuy, M., Phillips, M. M., Suntzeff, N. B., et al. 1996, AJ, 112, 2408

Hamuy, M., \& Pinto, P. A. 2002, ApJL, 566, L63

Hamuy, M., Pinto, P. A., Maza, J., et al. 2001, ApJ, 558, 615

Hamuy, M., \& Roth, M. 2003, IAUC, 8198, 3

Hamuy, M., Shectman, S., \& Thompson, I. 2002, IAUC, 8001, 2

Hamuy, M., \& Suntzeff, N. B. 1990, AJ, 99, 1146

Hamuy, M. A. 2001, PhD thesis, The Univ. Arizona

Heger, A., Fryer, C. L., Woosley, S. E., Langer, N., \& Hartmann, D. H. 2003, ApJ, 591, 288

Hendry, M. A., Smartt, S. J., Crockett, R. M., et al. 2006, MNRAS, 369, 1303 Holoien, T. W.-S., Prieto, J. L., Pejcha, O., et al. 2014, arXiv:1411.3322

Hurst, G. M., Armstrong, M., James, N., \& Foulkes, S. 1999, IAUC, 7275, 3 Hutchings, D., Li, W. D., \& Wood-Vasey, W. M. 2002, IAUC, 7964,

Inserra, C., Pastorello, A., Turatto, M., et al. 2013, A\&A, 555, A142

Inserra, C., Turatto, M., Pastorello, A., et al. 2012, MNRAS, 422, 1122

Ivanov, M. A., \& Shulman, G. A. 1990, SvA, 34, 163

Janka, H.-T. 2012, ARNPS, 62, 407

Janka, H.-T., Langanke, K., Marek, A., Martínez-Pinedo, G., \& Müller, B. 2007, PhR, 442, 38

Jerkstrand, A., Smartt, S. J., Sollerman, J., et al. 2015, MNRAS, 448, 2482

Jha, S., Challis, P., Garnavich, P., et al. 1999a, IAUC, 7296, 2

Jha, S., Garnavich, P., Challis, P., Kirshner, R., \& Berlind, P. 1999b, IAUC, 7280,2

Jones, M. I., Hamuy, M., Lira, P., et al. 2009, ApJ, 696, 1176

Kasen, D., \& Woosley, S. E. 2009, ApJ, 703, 2205

King, J. Y. 1999, IAUC, 7141, 1

Kirshner, R., \& Silverman, J. 2003, IAUC, 8042, 2

Kleiser, I., Poznanski, D., Kasen, D., et al. 2011, MNRAS, 415, 372

Klotz, A., Puckett, T., Langoussis, A., et al. 2002, IAUC, 7986, 1

Krisciunas, K., Hamuy, M., Suntzeff, N. B., et al. 2009, AJ, 137, 34

Leonard, D. C., Filippenko, A. V., Li, W., et al. 2002, AJ, 124, 2490

Li, W., Puckett, T., Kerns, B., \& Marcus, M. 2003, IAUC, 8214, 1

Li, W. D. 1999, IAUC, 7294, 1

Liu, Q.-Z., Hu, J.-Y., Hang, H.-R., et al. 2000, A\&AS, 144, 219

Llapasset, J.-M., Yamaoka, H., \& Ayani, K. 2003, CBET, 48, 1

Lloyd Evans, T., Evans, R., \& McNaught, R. H. 1986, IAUC, 4262, 2

Matheson, T., Challis, P., Kirshner, R., \& Berlind, P. 2003a, IAUC, 8225, 2

Matheson, T., Challis, P., Kirshner, R., \& Calkins, M. 2002, IAUC, 8016, 3

Matheson, T., Challis, P., Kirshner, R., \& Calkins, M. 2003b, IAUC, 8134, 2

Matheson, T., Challis, P., Kirshner, R., Calkins, M., \& Berlind, P. 2003c, IAUC, 8136,2

Maza, J., Hamuy, M., Antezana, R., et al. 1999, IAUC, 7210, 1

Maza, J., Hamuy, M., Antezana, R., Valladares, G., \& Aviles, R. 1993a, IAUC, 5812,2

Maza, J., Hamuy, M., Antezana, R., Wells, L., \& Kim, Y.-C. 1992a, IAUC, 5499, 1

Maza, J., Hamuy, M., Valladares, G., et al. 1993b, IAUC, 5693, 1

Maza, J., Hamuy, M., Wischnjewsky, M., et al. 1992b, IAUC, 5496, 1

Mazzali, P. A., Deng, J., Hamuy, M., \& Nomoto, K. 2009, ApJ, 703, 1624

McNaught, R. H., Evans, R., Spyromilio, J., et al. 1992, IAUC, 5552, 1

Miknaitis, G., Miceli, A., Garg, A., et al. 2002, IAUC, 8020, 1

Misra, K., Pooley, D., Chandra, P., et al. 2007, MNRAS, 381, 280

Monard, L. A. G. 2002, IAUC, 7995, 2

Monard, L. A. G. 2003, IAUC, 8186, 2

Moore, M., \& Li, W. 2003, CBET, 40, 1

Moore, M., Li, W., \& Boles, T. 2003, IAUC, 8184, 2

Morrell, N., \& Hamuy, M. 2003, IAUC, 8203, 2

Nomoto, K. I., Iwamoto, K., \& Suzuki, T. 1995, PhR, 256, 173

Nugent, P., Sollivan, M., Ellis, R., et al. 2006, ApJ, 645, 841

Olivares, E. F., Hamuy, M., Pignata, G., et al. 2010, ApJ, 715, 833

Papenkova, M., \& Li, W. 2003, IAUC, 8044, 1

Papenkova, M., Li, W., Lotoss/Kait, et al. 2003, IAUC, 8143, 2

Papenkova, M., \& Li, W. D. 2000, IAUC, 7410, 1

Pastorello, A., Valenti, S., Zampieri, L., et al. 2009, MNRAS, 394, 2266

Pastorello, A., Zampieri, L., Turatto, M., et al. 2004, MNRAS, 347, 74

Patat, F., Maza, J., Benetti, S., \& Cappellaro, E. 1999, IAUC, 7160, 2

Pejcha, O., \& Prieto, J. L. 2015a, ApJ, 799, 215

Pejcha, O., \& Prieto, J. L. 2015b, ApJ, 806, 225

Pennypacker, C. R., Burns, M. S., Crawford, F. S., et al. 1989, AJ, 97, 186

Phillips, M., \& Hamuy, M. 2003, IAUC, 8130, 4

Phillips, M., Hamuy, M., Roth, M., \& Morrell, N. 2003, IAUC, 8086, 2

Phillips, M., Maza, J., Antezana, R., et al. 1992, IAUC, 5570, 2

Phillips, M. M. 1992, IAUC, 5521, 1

Phillips, M. M. 1993a, IAUC, 5699, 2

Phillips, M. M. 1993b, ApJL, 413, L105 
Phillips, M. M., Hamuy, M., Heathcote, S. R., Suntzeff, N. B., \& Kirhakos, S. 1990, AJ, 99, 1133

Planck Collaboration, Ade, P. A. R., Aghanim, N., et al. 2015, arXiv: 1502.01589

Popov, D. V. 1993, ApJ, 414, 712

Poznanski, D., Butler, N., Filippenko, A. V., et al. 2009, ApJ, 694, 1067

Poznanski, D., Gal-Yam, A., Sharon, K., et al. 2003, IAUC, 8058, 1

Pozzo, M., Meikle, W. P. S., Rayner, J. T., et al. 2006, MNRAS, 368, 1169

Prieto, J. L., Lee, J. C., Drake, A. J., et al. 2012, ApJ, 745, 70

Pritchard, T. A., Roming, P. W. A., Brown, P. J., Bayless, A. J., \& Frey, L. H. 2014, ApJ, 787, 157

Puckett, T., Toth, D., Schwartz, M., et al. 2003, IAUC, 8117, 1

Revnivtsev, M., Tuerler, M., Del Santo, M., et al. 2003, IAUC, 8097, 2

Richmond, M. W. 2014, JAVSO, 42, 333

Rodríguez, Ó, Clocchiatti, A., \& Hamuy, M. 2014, AJ, 148, 107

Salvo, M., Bessell, M., \& Schmidt, B. 2003a, IAUC, 8187, 1

Salvo, M., Schmidt, B., \& Tonry, J. 2003b, IAUC, 8098, 2

Sanders, N. E., Soderberg, A. M., Gezari, S., et al. 2015, ApJ, 799, 208

Schlafly, E. F., \& Finkbeiner, D. P. 2011, ApJ, 737, 103

Schlegel, E. M. 1990, MNRAS, 244, 269

Schmidt, B. P., Kirshner, R. P., \& Eastman, R. G. 1992, ApJ, 395, 366

Schmidt, B. P., Kirshner, R. P., Eastman, R. G., et al. 1994a, ApJ, 432, 42

Schmidt, B. P., Kirshner, R. P., Eastman, R. G., et al. 1994b, AJ, 107, 1444

Schmidt, B. P., Kirshner, R. P., Schild, R., et al. 1993, AJ, 105, 2236

Singer, D., Beutler, B., Swift, B., et al. 2003, IAUC, 8201, 1

Smartt, S. J. 2015, PASA, 32, 16

Spiro, S., Pastorello, A., Pumo, M. L., et al. 2014, MNRAS, 439, 2873

Stritzinger, M., Hamuy, M., Suntzeff, N. B., et al. 2002, AJ, 124, 2100

Stritzinger, M. D., Phillips, M. M., Boldt, L. N., et al. 2011, AJ, 142, 156

Sukhbold, T., Entl, T., Woosley, S. E., Brown, J. M., \& Janka, H. T. 2015, arXiv: 1510.04643

Swift, B., Weisz, D., Li, W., \& Boles, T. 2003, IAUC, 8086, 1

Taddia, F., Stritzinger, M. D., Sollerman, J., et al. 2013, A\&A, 555, A10

Takáts, K., Pignata, G., Pumo, M. L., et al. 2015, MNRAS, 450, 3137

Tomasella, L., Cappellaro, E., Fraser, M., et al. 2013, MNRAS, 434, 1636
Tsvetkov, D. Y. 1994, AstL, 20, 374

Tsvetkov, D. Y. 2006, PZ, 26, 3

Tsvetkov, D. Y. 2008, PZ, 28, 3

Tsvetkov, D. Y., Goranskij, V., \& Pavlyuk, N. 2008, PZ, 28, 8

Tsvetkov, D. Y., Muminov, M., Burkhanov, O., \& Kahharov, B. 2007, PZ, 27,5

Tsvetkov, D. Y., Volnova, A. A., Shulga, A. P., et al. 2006, A\&A, 460, 769

Turatto, M., Cappellaro, E., Benetti, S., \& Danziger, I. J. 1993, MNRAS, 265,471

Valenti, S., Sand, D., Pastorello, A., et al. 2014, MNRAS, 438, L101

Vinkó, J., Takáts, K., Sárneczky, K., et al. 2006, MNRAS, 369, 1780

Weisz, D., \& Li, W. 2003, IAUC, 8131, 1

Wells, L., Maza, J., Antezana, R., et al. 1992, IAUC, 5554, 1

Wells, L., Maza, J., Wischnjewsky, M., et al. 1991, IAUC, 5310, 1

Williams, A., Martin, R., Schmidtke, P. C., et al. 1993, IAUC, 5733, 1

Winzer, J. E. 1974, JRASC, 68, 36

Wood, R., \& Andrews, P. J. 1974, MNRAS, 167, 13

Woodings, S., Martin, R., Williams, A., Verveer, A., \& Biggs, J. 1999, IAUC, 7158,1

Wood-Vasey, W. M., Aldering, G., Lee, B. C., et al. 2004, NewAR, 48, 637

Wood-Vasey, W. M., Aldering, G., \& Nugent, P. 2003a, IAUC, 8105, 1

Wood-Vasey, W. M., Aldering, G., \& Nugent, P. 2003b, IAUC, 8104, 2

Wood-Vasey, W. M., Aldering, G., Nugent, P., \& Chassagne, R. 2003c, IAUC, 8082,1

Wood-Vasey, W. M., Aldering, G., Nugent, P., \& Li, K. 2002a, IAUC, 8006, 3

Wood-Vasey, W. M., Aldering, G., Nugent, P., Mulchaey, J., \& Phillips, M. 2003d, IAUC, 8088, 2

Wood-Vasey, W. M., Aldering, G., Nugent, P., Papenkova, M., \& Li, W. 2003e, IAUC, 8101, 2

Wood-Vasey, W. M., Farris, B., Weisz, D., \& Li, W. D. 2002b, IAUC, 7967, 1

Woosley, S. E., Langer, N., \& Weaver, T. A. 1993, ApJ, 411, 823

Zampieri, L., Pastorello, A., Turatto, M., et al. 2003, MNRAS, 338, 711

Zhang, J., Wang, X., Mazzali, P. A., et al. 2014, ApJ, 797, 5

Zhang, T., Wang, X., Li, W., et al. 2006, AJ, 131, 2245 